\title{
Existence Theorems for Boundary Value Problems for Strongly Nonlinear Elliptic Systems
}

\author{
Hôǹg Thải Nguyêñ
}

\begin{abstract}
Let $L$ be a linear elliptic, a pseudomonotone or a generalized monotone operator (in the sense of F. E. Browder and I. V. Skrypnik), and let $F$ be the nonlinear Nemytskij superposition operator generated by a vector-valued function $f$. We give two general existence theorems for solutions of boundary value problems for the equation $L x=F x$. These theorems are based on a new functional-theoretic approach to the pair $(L, F)$, on the one hand, and on recent results on the operator $F$, on the other hand. We treat the above mentioned problems in the case of strong non-linearity $F$, i.e. in the case of lack of compactness of the operator $L-F$. In particular, we do not impose the usual growth conditions on the nonlinear function $f$; this allows us to treat elliptic systems with rapidly growing coefficients or exponential nonlinearities. Concerning solutions, we consider existence in the classical weak sense, in the socalled $L_{\infty}$-weakened sense in both Sobolev and Sobolev-Orlicz spaces, and in a generalized weak sense in Sobolev-type spaces which are modelled by means of Banach $L_{\infty}$-modules. Finally, we illustrate the abstract results by some applied problems occuring in nonlinear mechanics.

Keywords: Strongly nonlinear boundary value problems, existence theorems, solution in the usual weak sense, solution in the $L_{\infty}$-weakened sense, elliptic operators, pseudomonotone operators, generalized monotone operators, complementary systems, Sobolev spaces, Sobolev-Orlicz spaces, exact embedding theorems
\end{abstract}

AMS subject classification: Primary $35 \mathrm{~J} 65$, secondary $46 \mathrm{E} 30,47 \mathrm{H} 10,47 \mathrm{H} 17,47 \mathrm{H} 30$

\section{Introduction}

The present paper is concerned with the existence of solutions of strongly nonlinear elliptic boundary value problems, i.e. boundary value problems for elliptic equations and systems of the form.

$$
L x=F x
$$

on an open bounded subset $\Omega$ of $\mathbb{R}^{N}(N \geq 2)$, where $L$ is a linear or nonlinear elliptic differential operator of order $2 k$ in the divergence form

$$
\dot{L} x=\sum_{|\alpha| \leq k}(-1)^{|\alpha|} D^{\alpha} a_{a}\left(s, \delta_{k} x(s)\right)
$$

Hōng Thái Nguyêñ̃: Szczecin Univ., Inst. Math., ul. Wielkopolska 15, 70-451 Szczecin, Poland nguyenht@sus.univ.szczecin.pl.

nhthaimt@uoo.univ.szczecin.pl (November-June) and nguyenht@his-hu.ac.vn (July-October) 
with coefficients $a_{\alpha}$ satisfying conditions (including growth conditions) which guarantee the solvability of the problem $L_{x}=h$. The Carathéodory function $f(s, u): \Omega \times \mathbb{R}^{m} \rightarrow$ $\mathbb{R}^{m}$ generating the nonlinear Nemytskij superposition operator $F$ (see, e.g., $[3-4]$ ) where

$$
F x(s)=f(s, x(s))
$$

satisfies some condition but has otherwise completely inrestricted growth ("strongly nonlinear" growth) with respect to $u \in \mathbb{R}^{m}$. In other words, the "strong non-linearity" of $F$ means that we have to consider the cases of the operator $L-F$ with "lack of compactness" and of "critical exponents" in the "exact" Sobolev embedding theorems (S. L. Sobolev, S. I. Pokhozaev and N. S. Trudinger and their generalizations). The nonlinear function $f$ is assumed in the present paper to satisfy a general one-sided estimate, which covers all the so-called "sign condition" and "generalized sign condition". We point out that the above one-sided estimate is not sufficient for getting existence results in the non-scalar vector case $m>1$ (i.e. the case of systems of equations), and so for this case we should suppose some addittional conditions on the growth of $f$. This one-sided estimate, in particular, "compensates" the lack of both compactness and monotonicity type conditions of $L-F$.

Such problems in the scalar case (i.e. $m=1$ ) were first considered by F. E. Browder [9] and later on up to the present time by P. Hess, H. Brezis and F. E. Browder, J. Webb, J.-P. Gossez and others (see, for example, $[6-8,10,15-16,18,23,34]$, and the literature cited therein) via variational and topological methods.

In this paper we shall present two general existence theorems (see Theorem 1 in Section 7 and Theorem 2 in Section 8) following ideas of our dissertation [26] for problem (1). We consider both systems with polynomial growth coefficients and with exponential growth coefficients, as well as, both the existences in the usual weak sense and in the so-called $L_{\infty}$-weakened sense of $\mathrm{H}$. Brezis and F. E. Browder in Sobolev spaces and of J.$P$. Gossez in Sobolev-Orlicz spaces, and in more general spaces as so-called generalized Sobolev spaces (which are modelled by means of Banach function $L_{\infty}$-modules).

These theorems are based, on the one hand, on a new function-theoretic approach to the pair $(L, F)$, which unifies all the approaches of $[7-10,15-16]$, and the approaches $[2,26,37]$, and which is applicable not only to linear strongly elliptic operators $L$ or pseudomonotone operators $L$ but also to generalized monotone operators $L$ in the sense of Browder and Skrypnik (see, e.g., $[29,31-32]$ ) and, on the other hand, on the recent theories of Banach function,$L_{\infty}$-modules of vector-valued functions and of nonlinear operators acting in such spaces, which are presented in $[3-4,25-27,36]$. We point out that the structure of such spaces is much more complicated than in the scalar case, and thus the results presented here are not just generalizations from scalar differential equations to systems.

The plan of our paper is the following one. In Section 2 we shall give simplest variants of the two main theorems, namely Theorem 1 in Section 7 and Theorem 2 in Section 8 for the quasilinear elliptic boundary value problem (1) where the nonlinear superposition operator $F$ is a "strongly non-linearity" and $L$ is a operator, which is weakly nonlinear in the sense of M. I. Vishik - Yu. A. Dubinskij (see, e.g., $[11])[L \in \mathfrak{C}(\mathcal{L})]$, pseudomonotone in the sense of $\mathrm{H}$. Brezis $[L \in \mathfrak{C}(B M)]$ (in particular, a "variational operator" of J. Leray-J.-L. Lions) (sec, e.g., [21]), generalized monotone in the sense of 
F. E. Browder - I. V. Skrypnik (see, e.g., $[29,31$ - 32]) $[L \in \mathfrak{C}(B S)]$, or pseudomonotone with respect to complementary systems in the sense of J.-P. Gossez (see, e.g., [14 - 15, 17]) $[L \in \mathfrak{C}(G M)]$.

For these Theorems we shall give the definition of a complementary system

$$
\left(V, V_{0} ; W, W_{0}\right)
$$

in Section 3, introduce the classes of spaces $3 v(L, F, \mathfrak{F})$ in Section 4 (connected with onesided estimates and the strong non-linearity of $F$ ), $\mathfrak{N}(L, F)$ (with the exact definitions of the classes $\mathfrak{C}(\mathcal{L}), \mathfrak{C}(B M), \mathfrak{C}(B S), \mathfrak{C}(G M))$ in Section 5, condition (R1) in Lemma 3 and condition (R2) in Lemma 4 in Section $6,3 v(L, F, \Gamma)$ and $\mathfrak{N}(L, F, \Gamma)$ and $\operatorname{Tr}\left(V_{0}, \Gamma\right)$ in Section 8, and finally give some applications in nonlinear mechanics in Section 9.

We point out that these theorems can be extended also to variational elliptic inequalities as well as in many papers cited in [17], and we shall treat in detail this in a forthcoming paper.

\section{Some terminology and notations}

For our analysis in this paper we need the following notions concerning Banach spaces of measurable functions, in particular, the class of Banach $L_{\infty}$-modules (see [25 - 27, 36]).

Recall [36] that a Banach space $X$ of measurable functions $x: \Omega \rightarrow \mathbb{R}^{m}$ with norm $\|\cdot\|_{X}$ is called a Banach $L_{\infty}$-module (Banach function $L_{\infty}$-module) if $x \in X$ and $\alpha \in L_{\infty}$ imply that $\alpha x \in X$ and $\|\alpha x\|_{X} \leq\|\alpha\|_{L_{\infty}}\|x\|_{X}$. Here $L_{\infty}=L_{\infty}(\Omega, \mathbb{R})$ is the Banach algebra of all essentially bounded measurable scalar functions defined on $\Omega$. In the scalar case $m=1$, Banach $L_{\infty}$-modules are just Banach lattices with monotone norm (see, e.g., $[5,22,33]$ ). In the vector case $m>1$, the theory of Banach $L_{\infty}$-modules is more involved and requires tools from Convex and Set-Valued Analysis (see [25 - 26]). A prominent example of a Banach $L_{\infty}$-module is the Orlicz space $L_{M}=L_{M}\left(\Omega, \mathbb{R}^{m}\right)$ with the Luxemburg norm

$$
\|x\|_{L_{M}}=\inf \left\{\lambda>0: \int_{\Omega} M\left(s, \frac{x(s)}{\lambda}\right) d s<\infty\right\}
$$

where $M: \Omega \times \mathbb{R}^{m} \rightarrow[0, \infty]$ is a given Young funtion (see, e.g., $[5,24-25,30]$ ). If $M(s, u)=\|u\|^{p} \quad(1 \leq p<\infty)$, the Orlicz space $L_{M}$ is just the Lebesgue space $L_{p}$. Other important examples of Banach $L_{\infty}$-modules are the Lorentz space and the Marcinkiewicz space which are modelled by means of $L_{M}$ (see, e.g., $[5,19]$ ).

Let $X$ be a Banach $L_{\infty}$-module with the property that there exists a sequence $\left\{x_{i}\right\}_{i \in N} \subset X$ such that the linear hull of $\left\{x_{i}(s)\right\}_{i \in N}$ coincides with $\mathbb{R}^{m}$. for almost all $s \in \Omega$. Then there exists the non-trivial so-called (Köthe) associate space $X^{\prime}$ of all measurable functions $x^{\prime}: \Omega \rightarrow \mathbb{R}^{m}$ for which the pairing of measurable functions

$$
\left\langle x, x^{\prime}\right\rangle=\int_{\Omega}\left(x(s), x^{\prime}(s)\right) d s
$$


is finite for every $x \in X$, where $(\cdot, \cdot)$ is the usual scalar product in $\mathbb{R}^{m}$. The space $X^{\prime}$ equipped with the natural norm

$$
\left\|x^{\prime}\right\|_{X^{\prime}}=\sup \left\{\left\langle x, x^{\prime}\right\rangle:\|x\|_{X} \leq 1\right\}
$$

is then also a Banach $L_{\infty}$-module. $X^{\prime}$ can be interpreted as closed subspace of all integral linear functionals of the dual space $X^{*}$. For example, if $X=L_{M}$, then $X^{\prime}$ is the Orlicz space $L_{M}$. generated by the associate Young function

$$
M^{*}(s, v)=\sup \left\{(u, v)-M(s, u): u \in \mathbb{R}^{m}\right\} .
$$

We have $\left(L_{p}\right)^{\prime}=L_{p^{\prime}}$, where $\frac{1}{p}+\frac{1}{p^{\prime}}=1 \quad(1 \leq p \leq \infty)$.

The regular part $X^{\circ}$ of a Banach $L_{\infty}$-module $X$ is defined as Banach $L_{\infty}$-module of all measurable function $x \in X$ with absolutely continuous [equi-continuous] norm, i.e.

$$
X^{\circ}=\left\{x \in X: \lim _{\operatorname{mes}(D) \rightarrow 0}\left\|P_{D} x\right\|_{X}=0\right\},
$$

where $P_{D}$ denotes the multiplication operator by the characteristic function $\chi_{D}$ of a measurable set $D$. If $X=X^{\circ}$, then $X$ is called a regular space. The space $X^{\prime}$ coincides with the dual space $X^{*}$ of $X$ if and only if $X$ is regular. The space $L_{M}$ is regular if and only if $M$ satisfies the $\Delta_{2}$-condition (see $[24,26,30]$ ). The space $L_{p}$ is regular if $p<\infty$, and is not regular if $p=\infty$.

The embedding $Y \subset X$ of Banach $L_{\infty}$-modules $X$ and $Y$ is called absolutely bounded if the unit ball of $Y$ is an absolutely bounded subset of $X$ (under other name, it has uniformly absolutely continuous [equi-continuous] norms), i.e.

$$
\lim _{\operatorname{mes}(D) \rightarrow 0} \sup _{\|x\|_{Y} \leq 1}\left\|P_{D} x\right\|_{X}=0
$$

For example, the embedding $L_{p} \subset L_{q}$ is absolutely bounded, if $p>q$.

Let a Banach $L_{\infty}$-module $\Gamma$ of the associate space $X^{\prime}$ be total on a Banach $L_{\infty}$ module $X$. Then, a subset $\mathfrak{N}$ of $X$ is called a $\Gamma$-weakly $[\sigma(X, \Gamma)$-weakly] absolutely bounded set if the condition

$$
\lim _{\operatorname{mes}(D) \rightarrow 0} \sup _{x \in \mathfrak{N}}\left\langle P_{D} x, z\right\rangle=0 \quad(z \in \Gamma)
$$

holds. Later on the symbols $\hookrightarrow$ or $\hookrightarrow \hookrightarrow$ denotes continuous imbedding or compact imbedding of corresponding spaces, respectively. Denote by $S\left(\Omega, \mathbb{R}^{m}\right)$ the metric space of all measurable functions $x: \Omega \rightarrow \mathbb{R}^{m}$, equipped with the metric convergence in measure. For every Banach $L_{\infty}$-module $X$ we have $X \hookrightarrow S\left(\Omega, \mathbb{R}^{m}\right)$.

Remark that the theory of the Nemytskij superposition operator $F$ acting in Banach $L_{\infty}$-modules and in Orlicz spaces is given in $[3-4,26]$ for the vector case $m>1$, and in [5] for the scalar case $m=1$. 


\section{Simplest variants of main existence theorems}

In this Section we present the simplest variants of the Main Existence Theorems 1 and 2.

First, we formulate the following simplest variant of Theorem 1 (for operators $L$ in $\mathfrak{C}(\mathcal{L}), \mathfrak{C}(B M)$ or $\mathfrak{C}(B S)$ ) on the existence of solutions for the boundary value problem (1) with $x \in V$ where $V$ with $\mathscr{W}_{p}^{k}(\Omega) \hookrightarrow V \hookrightarrow W_{p}^{k}(\Omega) \quad(1<p<\infty)$ is a reflexive

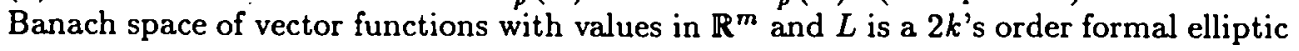
operator generating the operator $L: V \rightarrow V^{*}$. We denote by $Z$ a "minimal" Banach $L_{\infty}$-module such that $\mathrm{V}$ is continuously (but non-compactly) embedded into $Z$ (the imbedding inclusion $V \hookrightarrow Z$ is defined by means of Sobolev embedding theorems and their various generalizations).

Theorem 1'. Let $X, Y, \Gamma \subset S\left(\Omega, \mathbb{R}^{m}\right)$ be Banach $L_{\infty}$-modules, $Z \subset \Gamma, Z \subset X$ and $Z \subset Y$, and suppose that following conditions are satisfied:

(P1) $L: V \rightarrow V^{*}$ is a bounded operator, either hemicontinuous pseudomonotone in the sense of $H$. Brezis, or generalized monotone in the sense of $F$. E. Browder - I. V. Skrypnik.

(P2) There exists a Carathéodory function $H: \Omega \times \mathbb{R}^{m} \rightarrow[0, \infty)$ such that $(u, f(s, u))$ $\leq H(s, u)$ and $\langle L x, x\rangle-H[x]>0 \quad\left(\|x\|_{v} \geq r>0\right)$ for some $r>0$.

(P3) The embedding $Z \subset Y$ is absolutely bounded, and there exists a Carathéodory function $B: \Omega \times \mathbb{R}^{m} \rightarrow[0, \infty)$ such that $(u, f(s, u)) \leq B(s, u)$ and the superposition operator $B$ generated by the function $B(\cdot, \cdot)$ acts from $Y$ into $L(\Omega, \mathbb{R})$.

(P4) Either

(a) $m=1$ (the case of scalar equations) and $X=\Gamma$ and $F$ acts from $\Gamma$ into $\Gamma^{\prime}$ or

(b) $m>1$ (the case of systems of equations), $Z \subset X$ and $F: X \rightarrow \Gamma^{\prime}$ maps bounded subsets of $X$ into $\Gamma$-weakly absolutely bounded subsets of $\Gamma^{\prime}$

or else

(c) $m>1$ (the case of systems of equations), the imbedding $Z \subset X$ is absolutely bounded and $F$ acts from $X$ into $\Gamma^{\prime}$.

Then problem (1) has at least one solution in $V \subset X$.

We point out that in the case $L \in \mathfrak{C}(\mathcal{L}$ ) (in particular, in the case of a linear operator $L$ ) we can drop condition (P3). Then we get results containing as particular cases existence theorems obtained in [2] via other methods.

Second, we formulate the following simplest variant of Theorem 2 on the existence of solutions in the $\Gamma$-weakened sense for problem (1) where $L \in \mathfrak{C}(B M)$ or $L \in \mathfrak{C}(G M)$ ). We formulate this variant of Theorem 2 for the case when $L \in \mathfrak{C}(B M), \Gamma=L_{\infty}$ and $V=\dot{W}_{p}^{k}(\Omega)(1<p<\infty)$ that corresponds to the Dirichlet problem. Let $L: \dot{W}_{p}^{k}(\Omega) \rightarrow$ $W_{p^{\prime}}^{-k}(\Omega)$ be a $2 k$ 's order elliptic operator, $p^{\prime}=\frac{p}{p-1}$. We recall that the boundary value problem

$$
L x=F x \quad\left(x \in \dot{W}_{p}^{k}(\Omega)\right)
$$


is solvable in the $L_{\infty}$-weakened sense if there exists a function $x * \in \dot{W}_{p}^{k}(\Omega)$ such that

$$
\begin{aligned}
\left\langle y, L x_{*}\right\rangle_{V} & =\left\langle y, F x_{*}\right\rangle \quad\left(y \in L_{\infty} \cap \dot{W}_{p}^{k}(\Omega)\right) \\
\left\langle x_{*}, L x_{*}\right\rangle_{V} & =\left\langle x_{*}, F x_{*}\right\rangle .
\end{aligned}
$$

Let $X, Y \subset S\left(\Omega, \mathbb{R}^{m}\right)$ be Banach $L_{\infty}$-modules, $Z \subset X$ and $Z \subset Y$, where $Z=L_{p^{*}}$. if $k p<N, Z=L_{\infty}$ if $k p>N$, and $Z=L_{\phi}^{\circ}$ if $k p=N$ (here $p^{*}=\frac{N p}{N-k p}$ and $\left.\phi(s, u)=\exp \left(\|u\|^{\frac{p}{p-1}}\right)-1\right)$. By the exact embedding theorems of S. L. Sobolev (see [1]) and of S. I. Pokhozaev - N. S. Trudinger (see [1]) $\mathscr{W}_{p}^{k}(\Omega)$ is continuously (but noncompactly if $k p \leq N$ ) embedded into $Z$. Remember that $L_{\phi}^{\circ}$ is the regular part of the Orlicz space $L_{\phi}$ (equivalently, it is the closure of the set of continuous functions in $L_{\phi}$ ).

Theorem 2'. Suppose that the mentioned above conditions (P2) - (P4) for $\Gamma=L_{\infty}$ but without $Z \subset \Gamma$, and the following condition

$\left(\mathbf{P} 1^{\prime}\right) L: \dot{W}_{p}^{k}(\Omega) \rightarrow W_{p^{\prime}}^{-k}(\Omega)$ is an operator, which is bounded hemicontinuous and pseudomonotone in the sense of $H$. Brezis

are satisfied. Then problem (4) has at least one solution $x_{*}$ in the $L_{\infty}$-weakened sense (5) $-(6)$.

We point out that by the scheme for $L_{\infty}$-weakened solutions in the papers of $F$. E. Browder, F. E. Browder and H. Brezis, P. Hess, J. Webb, J.-P. Gossez, V. Mustonen, and others mathematicians (see, e.g., $[6-10,15-16,18,34]$ and the literature cited therein) one can consider only the case when $m=1$ and $H(s, u)=B(s, u)=b(s)$ with $b \in L(\Omega, \mathbb{R})$ (i.e. when the so-called "sign" condition $(u, f(s, u)) \leq b(s)$ is satisfied). We would like to point out that this "sign" condition turns out not to be sufficient for the existence of solutions in the non-scalar vector case $m \neq 1$ (i.e. the case of systems of equations), and so for this case we should suppose some addittional conditions on the growth of $f$ as in the above condition (P4)/(b) and (c).

\section{Elliptic differential operator $L$ and complementary system $\left(\mathbf{V}, \mathbf{V}_{\mathbf{0}} ; \mathbf{W}, \mathbf{W}_{\mathbf{0}}\right)$}

In this section we give the definition of an elliptic differential operator $L$ in a complementary system $\left(V, V_{0} ; W, W_{0}\right)$ and some facts about the construction of complementary system in applications.

Let $\Omega$ be a bounded open subset in the Euclidean space $\mathbb{R}^{N}(N \geq 2) ; \alpha=$ $\left(\alpha_{1}, \ldots, \alpha_{N}\right)$ a multi-index with non-negative natural components, $|\alpha|=\sum_{j}^{N} \alpha_{j}$. If $x=x(s)=\left(x_{1}(s), \ldots, x_{m}(s)\right)$ is some vector-valued function, then denote

$$
D^{\alpha} x=\frac{\partial^{|\alpha|} x}{\partial s_{1}^{\alpha_{1}} \cdots \partial s_{N^{\alpha}}}, \quad \delta_{k} x=\left\{D^{\alpha} x_{j}\right\}_{|a| \leq k}^{j \leq m}, \quad k(N)=\frac{(N+k) !}{N ! k !} .
$$

Let $L$ be a $2 k$ 's order elliptic differential operator in the divergence form (2) where $a_{\alpha}: \Omega \times \mathbb{R}^{m k(N)} \rightarrow \mathbb{R}^{m}$ are Carathéodory functions, i.e. $a_{\alpha}(\cdot, u)$ is measurable for all $u$ and $a_{\alpha}(s, \cdot)$ is continuous for almost all $s$. 
Let $V$ and $W$ be two Banach spaces, $V$ of vector-valued functions $x: \Omega \rightarrow \mathbb{R}^{m}$ such that $x$ and its distribution partial derivatives up to order $k$ lie in $S\left(\Omega, \mathbb{R}^{m}\right)$. Suppose that $(V, W)$ is a duality pairing with respect to a bilinear form $\langle\cdot, \cdot\rangle_{v}$ for which

$$
\langle\dot{x}, y\rangle_{V}=\langle x, y\rangle \quad\left(x \in V \subset S\left(\Omega, \mathbb{R}^{m}\right), y \in W \cap S\left(\Omega, \mathbb{R}^{m}\right)\right)
$$

holds. Further, let

$$
\left(V, V_{0} ; W, W_{0}\right)
$$

be a complementary system in the sense of T. Donaldson - J.-P. Gossez [13 - 14], i.e. $V_{0} \subset V$ and $W_{0} \subset W$ are subspaces such that, by means of $\langle\cdot, \cdot\rangle_{V}, V_{0}^{*}$ can be identified with (i.e. is linear homeomorphic to) $W$ and $W_{0}^{*}$ with $V$.

Suppose that the formal differential operator (2) defines the operator $L: V \rightarrow$ $W$ with domain $D(L) \supset V_{0}$ (which, in general cases, is not everywhere defined and nonlinear) by

$$
\langle y, L x\rangle_{V}=L(x, y) \quad\left(y \in V_{0}, x \in D(L)\right)
$$

and

$$
L(x, y)=\int_{\Omega} \sum_{|\alpha| \leq k}\left(a_{\alpha}\left(s, \delta_{k} x(s)\right), D^{\alpha} y(s)\right) d s .
$$

We point out that in applications for the case of reflexive Banach spaces $V$ we shall use the complementary system $\left(V, V ; V^{*}, V^{*}\right)$. In applications for the non-refexive case we shall use for the Dirichlet problem the system

$$
\left(\stackrel{\circ}{W}^{k} E, \stackrel{\circ}{W}^{k} E^{\circ} ; W^{-k} E^{\prime}, W^{-k}\left(E^{\prime}\right)^{\circ}\right)
$$

and for the Neumann problem the system

$$
\left(V, V_{0} ; W, W_{0}\right) \quad \text { with } V=W^{k} E \text { and } V_{0}=W^{k} E^{\circ}
$$

(see references in $[13-14,17,19])$. Here $E \subset S\left(\Omega, \mathbb{R}^{m k(N)}\right)$ is a Banach $L_{\infty}$-module and $E^{\circ}$ is its regular part (see Section 1 ), and $W^{k} E$ is the "generalized" Sobolev-like space (modelled by means of $E$ ) of vector-valued functions $x$ with the norm

$$
\|x\|_{W^{k} E}=\left\|\delta_{k} x\right\|_{E}
$$

( $\delta_{k} x$ is the vector of which components are all distribution partial derivatives of $x$ up to order $k$ ), and $W^{k} E$ is the $\sigma\left(E,\left(E^{\prime}\right)^{\circ}\right)$-weak closure of $C_{\circ}^{\infty}\left(\Omega, \mathbb{R}^{m}\right)$ in $W^{k} E$. Remember (see, e.g., $[13-14,21]$ ) that $V$ with $\stackrel{\circ}{W}_{p}^{k} \subset V \subset W_{p}^{k}$ (the usual Sobolev spaces) corresponds to boundary value problems for equations having coefficients with polynomial growth, and $V$ with $\dot{W}^{k} L_{M} \subset V \subset W^{k} L_{M}$ (the Sobolev-Orlicz spaces) corresponds to boundary value problems for equations having coefficients. with rapidly or slowly increasing growths, etc. 


\section{Class $3 v(L, F, \mathfrak{F})$}

Let $f: \Omega \times \mathbb{R}^{m} \rightarrow \mathbb{R}^{m}$ be a Carathéodory function generating the superposition operator $F: V \rightarrow W$ and having domain $D(F)$. Sections $4-8$ devote the existence investigation on the boundary value problem (1) where $L: V \rightarrow W$ is the operator with domain $D(L)$ which was introduced in Section 3.

We put

$$
T_{V}(r)=\left\{x \in V:\|x\|_{V} \leq r\right\} \quad(0<r<\infty)
$$

and

$$
H(r)=\left\{L x: x \in T_{V}(r), L x=\theta F x \text { for some } \theta \in \Xi\right\}
$$

where $\Xi$ is the set of all measurable scalar functions such that $0 \leq \theta(s) \leq 1$ for almost all $s \in \Omega$. We recall that $(Y, \Gamma) \in 3 v(L, F, \mathcal{F})$ (here $\Gamma$ is a total on a Banach $L_{\infty}$-module $Y$ Banach $L_{\infty}$-submodule of $\left.Y^{\prime}\right)$ if the sets $H(r) \quad(0<r<\infty)$ are $\Gamma$-weakly absolutely bounded in $Y$.

The verification of the inclusion $(Y, \Gamma) \in 3 v(L, F, \mathcal{F})$ is connected with many considerable difficulties. We shall describe the main non-trivial scheme for this in the present section.

We shall be interested in the imbedding $V \hookrightarrow \hookrightarrow S\left(\Omega, \mathbb{R}^{m}\right)$ and in constructing a Banach $L_{\infty}$-module $X$ such that $V \hookrightarrow X$ or $V \hookrightarrow \hookrightarrow X$. The imbedding $V \hookrightarrow X$ can be defined by embedding theorems of Sobolev type, and the imbedding $V \hookrightarrow \hookrightarrow S\left(\Omega, \mathbb{R}^{m}\right)$ follows from theorems of Rellich-Kondrashov type (see, e.g., $[1,19]$ and the various literature cited therein).

The verification of the imbedding $V \hookrightarrow \hookrightarrow X$ is connected with the following lemma.

Lemma 1. Let $X_{0}, X \subset S\left(\Omega, \mathbb{R}^{m}\right)$ be Banach $L_{\infty}$-modules, $V \hookrightarrow X_{0}, V \hookrightarrow \hookrightarrow$ $S\left(\Omega, \mathbb{R}^{m}\right)$ and $X_{0} \subset X$ be an absolutely bounded embedding. Then $V \hookrightarrow \hookrightarrow X$ holds.

Proof. By assumptions the unit ball of $V$ is pre-compact in $S\left(\Omega, \mathbb{R}^{m}\right)$ and absolutely bounded in the Banach $L_{\infty}$-module $X$. Hence, it is pre-compact in $X$ by [27: Theorem 8]

We recall that $(L, F) \in \mathcal{P}_{\mathrm{o}}(V, Z)$ (where $Z$ is a Banach $L_{\infty}$-module and $V \hookrightarrow Z$ ) if $f$ satisfies the inequalities

$$
(\pi u, f(s, u)) \leq A_{\pi}(s, u) \geq 0 \quad\left(A_{\pi}(s, 0)=0\right)
$$

and if the inequalities

$$
\begin{aligned}
& \left\|A_{\pi} x\right\|_{L} \leq a_{\pi}(r) \quad\left(\|x\|_{Z} \leq r, \pi \in \mathcal{P}\right) \\
& \langle\pi x, L x\rangle_{V} \geq c_{\pi}(r) \quad\left(\pi \in \mathcal{P}, x \in T_{V}(r) \cap D(L)\right)
\end{aligned}
$$

hold where $a_{\pi}(r)$ and $c_{\pi}(r)(r>0)$ are constants, and $A_{\pi}: \Omega \times \mathbb{R}^{m} \rightarrow \mathbb{R}$ are superpositionally measurable (for example, Carathéodory) functions generating the superposition operators $A_{\pi}$, and $\mathcal{P}=\{\pi\}$ is some (maybe, empty) family of orthogonal projectors in $\mathbb{R}^{m}$ which commute with operators $L$ and $F$. In applications, $\mathcal{P}=\{\emptyset\}, \mathcal{P}=\{I\}$ with 
$I$ the identity operator or $\mathcal{P}=\{\cdot j: j=1, \ldots, m\}$ where,$j$ is the operator of passing to the $j$ 'th component.

We put

$$
\Delta\left(d_{\pi}\right)=\left\{L x: x \in D(L), \int_{\Omega}|(\pi x(s), L x(s))| d s \leq d_{\pi}\right\} \quad(\pi \in \mathcal{P}) .
$$

Lemma 2. Let $(L, F) \in \mathcal{P}_{\mathrm{o}}(V, Z)$. Then $H(r) \subset \Delta\left(d_{\pi}\right)$ where $d_{\pi}=2 a_{\pi}(c r)-$ $c_{\pi}(r)(\pi \in \mathcal{P})$ and $c=c(V, Z)$ is the imbedding constant of $V$ into $Z$.

Proof. Assume $y \in H(r)$. Then for some $x \in T_{V}(r) \cap D(L)$ and for some $\theta \in \Xi$, $y=L x=\theta F x$. Evidently,

$$
\int_{\Omega}(\pi x, \theta F x) d s=\int_{\Omega}(\pi x, L x) d s=\langle\pi x, L x\rangle \geq c_{\pi}(r) .
$$

For each measurable set $D$ we have

$$
\int_{D}(\pi x, \theta F x) d s \leq \int_{D} A_{\pi} x(s) d s=\left\|A_{\pi}\left(P_{D} x\right)\right\|_{L} \leq a_{\pi}(c r) .
$$

Let $\Omega(\pi)$ denote the set of all $s \in \Omega$ such that $(x(s), \theta(s) F x(s)) \geq 0$. Then

$$
\begin{aligned}
\int_{\Omega}|(\pi x, L x)| d s & =\int_{\Omega}|(\pi x, \theta F x)| d s=\left(2 \int_{\Omega(\pi)}-\int_{\Omega}\right)(\pi x, \theta F x) d s \\
& \leq d_{\pi}=2 a_{\pi}(c r)-c_{\pi}(r)=d_{\pi}(r)
\end{aligned}
$$

and the lemma is proved

Now suppose that $f$ satisfies else the inequality (in this case we write $f \in \mathcal{P}^{\circ}(V)$ )

$$
(v, f(s, u)) \leq \sum_{\pi \in \mathcal{P}} c_{\pi}|(\pi u, f(s, u))|+P(s, u)+Q(s, v)+R(s, v, u)
$$

where $c_{\pi}(\pi \in \mathcal{P})$ are non-negative constants and $P, Q, R(R(s, v, 0)=0)$ are Carathédory functions such that the corresponding superposition operators $P, Q, R$ map $V, \Gamma, \Gamma \times$ $V$ into $L(\Omega, \mathbb{R})$, respectively, where $\Gamma$ is a total on $Y$ Banach $L_{\infty}$-module of $Y^{\prime}$.

Proposition 1. Assume $Y=\Gamma^{\prime},(L, F) \in \mathcal{P}_{\circ}(V, Z)$, and $f \in \mathcal{P}^{\circ}(V)$, and that one of the following conditions is satisfied:

(i) $V \hookrightarrow \hookrightarrow X$.

(ii) $V \hookrightarrow X$, the operator $P$ is bounded on each ball of $X$, and the operator $R(z, \cdot)$ is absolutely bounded on each ball of $X$ for every fixed $z \in \Gamma$.

(iii) $V \hookrightarrow X, \Gamma$ is a regular Banach $L_{\infty}$-module, i.e. $\Gamma=\Gamma^{\circ}$, and the operator $R(z, \cdot)$ is bounded on each ball of $X$ for every fixed $z \in \Gamma$.

Then $(Y, \Gamma) \in \mathfrak{Z} v(L, F, \mathcal{F})$. 
Proof. From Lemma 2 and (16) the inequality

$$
\left|\left\langle P_{D} z, \theta F x\right\rangle\right| \leq \lambda \sum_{\pi \in \mathcal{P}} c_{\pi} d_{\pi}(r)+\lambda\|P x\|_{L}+\lambda\left\|P_{D} Q\left(\lambda^{-1} z\right)\right\|_{L}+\lambda\left\|P_{D} R\left(\lambda^{-1} z, x\right)\right\|_{L}
$$

follows where $0<\lambda<\infty, z \in \Gamma, L x=\theta F x \in H(r), x \in T_{V}(r)$ and $\theta \in \Xi$. By (17) and the assumptions of Proposition 1 we have

$$
\begin{aligned}
\left|\left\langle P_{D} z, \theta F x\right\rangle\right| \leq & \lambda \sum_{\pi \in \mathcal{P}} c_{\pi} d_{\pi}(r)+\lambda \sup _{\|x\| x \leq c r}\|P x\|_{L} \\
& +\lambda\left\|P_{D} Q\left(\lambda^{-1} z\right)\right\|+\lambda \sup _{\substack{x \in T_{V}(r) \\
L x=0 F x}}\left\|P_{D} R\left(\lambda^{-1} z, x\right)\right\|_{L} \quad(0<\lambda<\infty)
\end{aligned}
$$

where $c$ is the imbedding constant of $V \hookrightarrow X$.

By either $V \hookrightarrow \hookrightarrow X$ or the boundedness of the superposition operator $P: X \rightarrow$ $L(\Omega, \mathbb{R})$ it follows that for every fixed $\varepsilon>0$ we can find a suitable number $\lambda_{\varepsilon}>0$ such that

$$
\left|\left\langle P_{D} z, \theta F x\right\rangle\right| \leq \varepsilon+\lambda_{e}\left\|P_{D} Q\left(\lambda_{\varepsilon}^{-1} z\right)\right\|+\lambda_{\varepsilon} \sup _{\substack{x \in T_{V}(r) \\ L x \in O F z}}\left\|P_{D} R\left(\lambda_{\varepsilon}^{-1} z, x\right)\right\|_{L} .
$$

By the Krasnoselskij type continuity theorem for superposition operators acting in Banach $L_{\infty}$-modules (see, e.g., [4: Theorem 3]) and of the Vitali-Krasnoselskij compactness criterion (see, e.g. [20] and [27: Theorem 8]) for subsets in $L(\Omega, \mathbb{R})$, in the case when assumption (i) or (ii) holds, the equality

$$
\lim _{\operatorname{mes}(D) \rightarrow 0} \sup _{\substack{x \in T_{V}(r) \\ L x=\theta F x}}\left|\left(P_{D^{z}} z, \theta F x\right\rangle\right|=0
$$

holds and, in the case when assumption (iii) holds, the inequality

$$
\sup _{\substack{x \in T_{V}(r) \\ L x=\theta F x}}|\langle z, \theta F x\rangle|<\infty
$$

holds which, by regularity of the Banach $L_{\infty}$-module $\Gamma$ and the Banach-Steinhaus theorem, is (see [26] and [27: Theorem 8]) equivalent to (18). Equality (18) means that the set $H(r)$ (see (12)) is $\Gamma$-weakly absolutely bounded in $Y$ for all $r>0$, i.e. $(Y, \Gamma) \in \mathfrak{Z}_{v}(L, F, \mathcal{F})$

As simple but important specializations of Proposition 1, we obtain the following two corollaries.

Corollary 1. Suppose $Y=\Gamma^{\prime}$ and suppose that one of the following conditions is satisfied:

(i) $V \hookrightarrow \hookrightarrow X$ and the operator $F$ acts from $X$ into $Y$.

(ii) $V \hookrightarrow X$, and the operator $F: X \rightarrow Y$ maps every bounded subset of $X$ into a $\Gamma$-weakly absolutely bounded subset of $Y$.

(iii) $V \hookrightarrow X, \Gamma$ is a regular Banach $L_{\infty}$-module, and the operator $F: X \rightarrow Y$ maps every bounded subset of $X$ into a $\Gamma$-weakly bounded subset of $Y$. 
Then $(Y, \Gamma) \in 3(L, F, \mathcal{F})$.

Proof. We have

$$
(v, f(s, u)) \leq R(s, v, u)
$$

where $R(s, v, u)=|(v, f(s, u))|$. As assumed, all assumptions of Proposition 1 hold for the case when $\mathcal{P}=\{\emptyset\}, P(s, u)=Q(s, v)=0$. Hence, from Proposition 1, Corollary 1 follows

Corollary 2. Assume $Y=\Gamma^{\prime}, m=1,(L, F) \in \mathcal{P}_{\mathrm{o}}(V, Z)$ and $\mathcal{P}=\{I\}$ with $I$ the identity operator. Suppose, moreover, that the superposition operator $F$ acts from $\Gamma$ into $Y$. Then $(Y, \Gamma) \in \mathcal{Z}(L, F, \mathcal{F})$ if $V \hookrightarrow \hookrightarrow S\left(\Omega, \mathbb{R}^{m}\right)$.

Proof. We set

$$
\tilde{f}(s ; u)=\sup \{|\cdot f(s, w)|:|w| \leq|u|\} .
$$

Then (see [4: Lemma 5], noting that coci $f=(-1,1] \tilde{f}) \tilde{f}$ will be also a Carathéodory function and the superposition operator $\tilde{f}$ acts from $\Gamma$ into $Y$, too. We have

$$
(v, f(s, u)) \leq|v||f(s, u)| \leq|u f(s, u)|+R(s, v, u)
$$

where $R(s, v, u)=|v| \tilde{f}(s, v)$. Hence, condition (1) of Proposition 1 follows in the case when $\mathcal{P}=\{I\}, c_{I}=1, P(s, u)=Q(s, v)=0$ and $X$ is an arbitrary Banach $L_{\infty^{-}}$ module such that the imbedding $Z \hookrightarrow X$ is absolutely bounded (and then, by Lemma $1, \dot{V} \hookrightarrow \hookrightarrow X)$. This proves that Corollary 2 follows from Proposition 1/(i)

In the end of Section 4 we remark that the condition $(Y, \Gamma) \in \mathfrak{Z}(L, F, \mathcal{F})$ is analogous to the so-called $k_{*}$-compactness and $b_{*}$-boundedness of the non-linearity $F$ in the sense of [35]. Via the schemes [35] and [28] one can find other complicated sufficient conditions which guarantee $(Y, \Gamma) \in 3 v(L, F, \mathcal{F})$, and, in particular, a vector analog of Corollary 2 by using $\mathcal{P}=\{. j: j=1, \ldots, m\}$ where.$j$ is the operator of passing to the $j$ 'th component.

\section{Classes $\mathfrak{N}(L, F), \mathfrak{C}(\mathcal{L}), \mathfrak{C}(B M), \mathfrak{C}(B S)$ and $\mathfrak{C}(G M)$}

We recall that $\left(V, V_{0} ; W, W_{0}\right) \in \mathfrak{N}(L, F)$ if for any sequences $\left\{x_{n}\right\} \subset D(L)$ and $\left\{\theta_{n}\right\} \subset \Xi$ such that

$L x_{n}=\theta_{n} F x_{n}$

$x_{n}$ converges to $x$ in the metric of $S\left(\Omega, \mathbb{R}^{m}\right)$

$x_{n}$ converges in the weak topology $\sigma\left(V, W_{0}\right)$,

$\theta_{n}$ converges to 1 in $S\left(\Omega, \mathbb{R}^{m}\right)$,

$L x_{n}$ converges to $\kappa$ in the weak topology $\sigma\left(W, V_{0}\right)$,

$L x=\kappa=F \dot{x}$ follows. Remember that, as before, $\Xi$ is the family of all measurable scalar functions $\theta$ such that $0 \leq \theta(s) \leq 1$ for a.a. $s \in \Omega$.

The verification of the inclusion $\left(V, V_{0} ; W, W_{0}\right) \in \mathfrak{N}(L, F)$ is connected with the following well-known concrete classes of operators $L$ and with the inclusion $(Y, \Gamma) \in$ $3_{v}(L, F, \mathcal{F})$. In this paper we use the classes $\mathfrak{C}(\mathcal{L}), \mathfrak{C}(B M), \mathfrak{C}(B S)$ and $\mathfrak{C}(G M)$ of $L$ which will be defined below. 
We recall (see Yu. A. Dubinskij [11], and also [31 - 32]) that $L \in \mathfrak{C}(\mathcal{L})$ if $V=V_{0}$ is a reflexive Banach space, $W=W_{0}=V^{*}$, and the operator $L: V \rightarrow W \quad(D(L)=V)$ is bounded and weakly continuous (i.e. $L x_{n}$ weakly converges to $L x$ if a generalized sequence $x_{n}$ converges weakly to $x$; in the case of a separable Banach space $V$ it is sufficient to consider a usual sequence $x_{n}$ ).

We recall (see [21] and Brezis's papers cited therein) that $L \in \mathfrak{C}(B M)$ if $V=V_{0}$ is a reflexive Banach space, $W=W_{0}=V^{*}$, the operator $L: V \rightarrow W(D(L)=V)$ is bounded, weakly continuous on every finite-dimensional subspace of $V$ and of the type $(\mathrm{M})$, i.e. has the following property (M): for every sequence $\left\{x_{n}\right\}$ such that $x_{n} \rightarrow x$ weakly, $L x_{n} \rightarrow \kappa$ weakly and lim $\sup _{n \rightarrow \infty}\left\langle x_{n}, L x_{n}\right\rangle_{V} \leq\langle x, \kappa\rangle_{V}$ it follows that $\kappa=L x$.

We recall (see, e.g., $[29,31-32,38]$ ) that $L \in \mathfrak{C}(B S)$ if $V=V_{0}$ is a reflexive Banach space, $W=W_{0}=V^{*}, L: V \rightarrow W(D(L)=V)$ is a bounded operator, which is demicontinuous (i.e. which maps every convergent in norm sequence into a weakly convergent sequence) and it has the Browder - Skrypnik property (so-called condition $\left.(S)_{+}\right)$: for any sequence $\left\{x_{n}\right\}$ such that $x_{n} \rightarrow x$ weakly and $\lim _{n}\left\langle x_{n}-x, L x_{n}\right\rangle_{V} \leq 0$ it follows that $x_{n} \rightarrow x$ in norm.

We recall (see J.-P. Gossez [13 - 14], and also [17]) that $L \in \mathfrak{C}(G M)$ if

1) $D(L) \supset V_{0}$ and $L$ is continuous on every finite-dimensional subspace of $V$ as an operator acting from $V$ into $\left(W, \sigma\left(W, V_{0}\right)\right)$

2) $L$ is sequentially pseudomonotone, i.e. for any sequence $\left\{x_{n}\right\} \subset D(L)$ such that $x_{n} \rightarrow x$ in the weak topology $\sigma\left(V, W_{0}\right), L x_{n} \rightarrow \kappa$ in the weak topology $\sigma\left(W, V_{0}\right)$ and $\limsup _{n \rightarrow \infty}\left\langle x_{n}, L x_{n}\right\rangle_{V} \leq\langle x, \kappa\rangle_{V}$ it follows that $x \in D(L), \kappa=L x$ and $\left\langle x_{n}, L x_{n}\right\rangle_{V}$ converges to $\langle x, \kappa\rangle_{V}$.

We recall that $V \in \mathfrak{N}(F)$ if for any sequences $\left\{x_{n}\right\}$ and $\left\{\theta_{n}\right\} \subset \Xi$ such that $x_{n} \rightarrow x$ in the weak topology $\sigma\left(V, W_{0}\right)$ and in $S\left(\Omega, \mathbb{R}^{m}\right), \theta_{n} \rightarrow 1$ in $S(\Omega, \mathbb{R})$ and $\left|\left(x_{n}, \theta_{n} F x_{n}\right\rangle\right|<\infty$, the inequality

$$
\underset{n \rightarrow \infty}{\limsup }\left\langle x_{n}, \theta_{n} F x_{n}\right\rangle \leq\langle x, F x\rangle<\infty
$$

follows.

Proposition 2. Suppose $(u, f(s, u)) \leq B(s, u) \geq 0$, with $B: \Omega \times \mathbb{R}^{m} \rightarrow \mathbb{R} a$ Carathéodory function generating the superposition operator $B: Z_{*} \rightarrow L(\Omega, \mathbb{R})$ where $V \hookrightarrow \hookrightarrow Z_{*}$ and $Z_{*} \subset S\left(\Omega, \mathbb{R}^{m}\right)$ is a Banach $L_{\infty}$-module. Then $V \in \mathfrak{N}(F)$.

Proof. Assume that $\left\{x_{n}\right\}$ and $\left\{\theta_{n}\right\}$ are sequences of functions in the definition of $V \in \mathfrak{N}(F)$. Then from $V \hookrightarrow \hookrightarrow Z_{*}$, it follows that $x_{n} \rightarrow x$ in the norm of $Z_{*}$. By the Krasnoselskij-type continuity theorem (see, e.g., [4: Theorem 3]), the superposition operator $B: Z * L(\Omega, \mathbb{R})$ is continuous, and consequently, $B x_{n} \rightarrow B x$ in $L(\Omega, \mathbb{R})$. By the Nemytskij theorem (see, e.g., in $[20]), \theta_{n} F x_{n} \rightarrow F x$ in $S\left(\Omega, \mathbb{R}^{m}\right)$.

Further, by $B x_{n}(s)-\left(x_{n}(s), \theta_{n} F x_{n}(s)\right) \geq 0$, from the Fatou lemma the inequality

$$
\liminf _{n \rightarrow \infty} \int_{\Omega}\left(B x_{n}(s)-\left(x_{n}(s), \theta_{n} F x_{n}(s)\right)\right) d s \geq \int_{\Omega}(B x-(x, F x)) d s
$$


follows. This inequality implies that

$$
\begin{aligned}
0 & \leq \int_{\Omega}(B x-(x, F x)) d s \\
& \leq \liminf _{n \rightarrow \infty} \int_{\Omega} B x_{n}(s) d s+\liminf _{n \rightarrow \infty} \int_{\Omega}\left(-\left(x_{n}, \theta_{n} F x_{n}\right)\right) d s \\
& =\int_{\Omega} B x(s) d s-\underset{n \rightarrow \infty}{\limsup } \int_{\Omega}\left(x_{n}, \theta_{n} F x_{n}\right) d s .
\end{aligned}
$$

Consequently,

$$
\limsup _{n \rightarrow \infty} \int_{\Omega}\left(x_{n}, \theta_{n} F x_{n}\right) d s \leq \int_{\Omega}(x, F x) d s \leq \int_{\Omega} B x(s) d s<\infty
$$

which proves (19)

Proposition 3. Assume $\Gamma^{\prime}=Y, V_{0} \hookrightarrow \Gamma$ and that one of the following conditions is satisfied:

(i) $L \in \mathfrak{C}(\mathcal{L}),(Y, \Gamma) \in 3 \vee(L, F, \mathcal{F})$.

(ii) $L \in \mathfrak{C}(B M),(Y, \Gamma) \in 3 v(L, F, \mathcal{F}), V \in \mathfrak{N}(F)$.

(iii) $L \in \mathfrak{C}(B S),(Y, \Gamma) \in \mathfrak{Z}_{v}(L, F, \mathcal{F}), V \in \mathfrak{N}(F)$.

(iv) $L \in \mathfrak{C}(G M),(Y, \Gamma) \in 3 v(L, F, \mathcal{F}), V \in \mathfrak{N}(F)$.

Then $\left(V, V_{0} ; W, W_{0}\right) \in \mathfrak{N}(L, F)$.

Proof. Let $x_{n}, \theta_{n}, \kappa, x$ be functions in the definition of $\mathfrak{N}(L, F)$. First, we consider the case when condition (i) is satisfied. Then, by $L \in \mathfrak{C}(\mathcal{L})$ we have at once the equality $L x=\kappa$. It remains to prove $\kappa=F x$. Since $\theta_{n} \rightarrow 1$ in $S(\Omega, \mathbb{R})$ and $x_{n} \rightarrow x$ in $S\left(\Omega, \mathbb{R}^{m}\right)$, then by the Nemytskij theorem (see, e.g., in $[5,20]) \theta_{n} F x_{n} \rightarrow F x$ in $S\left(\Omega, \mathbb{R}^{m}\right)$. Since $(Y, \Gamma) \in 3(L, F, \mathcal{F})$ and the set $\left\{x_{n}\right\}$ is bounded in $V$, the set $\left\{\theta_{n} F x_{n}\right\}$ is $\Gamma$-weakly absolutely bounded in $Y$. By the Vitali-Krasnoselskij convergence theorem (see, e.g., [20] and [27: Theorem 8]) in $L(\Omega, \mathbb{R})$,

$$
\left\langle z, \theta_{n} F x_{n}\right\rangle \rightarrow\langle z, F x\rangle \quad(z \in \Gamma)
$$

as $n \rightarrow \infty$ and therefore $\theta_{n} F x_{n} \rightarrow F x \Gamma$-weakly in $Y$. Since $V_{0} \hookrightarrow \Gamma$ and $\Gamma^{\prime}=Y$, the restriction of every functional $\langle\cdot, y)$ on $V_{0} \quad(y \in Y)$ is a element of $V_{0}^{*}=W$. By the definition of a complementary system $\left(V, V_{0} ; W, W_{0}\right)$ (see Section 3 ) it follows that $\left\langle z, \theta_{n} F x_{n}\right\rangle=\left\langle z, \theta_{n} F x_{n}\right\rangle_{V}$ and $\langle z, F x\rangle=\langle z, F x\rangle_{V} \quad\left(z \in V_{0}\right)$. From (21) it follows

$$
\left\langle z, \theta_{n} F x_{n}\right\rangle_{V} \rightarrow\langle z, F x\rangle_{V} \quad\left(z \in V_{0}\right),
$$

as $n \rightarrow \infty$, i.e. $\theta_{n} F x_{n}=L x_{n} \rightarrow F x$ in the weak topology $\sigma\left(W, V_{0}\right)$. Hence, $F x=\kappa$.

We are going to consider the case when condition (ii) is satisfied. As in the preceding case one can prove that $\theta_{n} F x_{n}=L x_{n} \rightarrow F x$ in the weak topology $\sigma\left(W, V_{0}\right)$ and $F x=\kappa$. It remains to prove that $L x=\kappa$. By $V \in \mathfrak{N}(F)$ we have (19). Then

$$
\limsup _{n \rightarrow \infty}\left\langle x_{n}, L x_{n}\right\rangle_{V}=\underset{n \rightarrow \infty}{\limsup }\left\langle x_{n}, \theta_{n} F x_{n}\right\rangle \leq\langle x, F x\rangle=\langle x, \kappa\rangle_{V}
$$


By property (M) for $L \in \mathfrak{C}(B M), L x=\kappa$ follows. Thus, $L x=\kappa=F x$.

Now we consider the case when condition (iii) is satisfied. As in the first case one can prove that $\theta_{n} F x_{n}=L x_{n} \rightarrow F x$ in the weak topology $\sigma\left(W, V_{0}\right)$ and $\kappa=F x$. It remains to prove that $L x=\kappa$. By $V \in \mathfrak{N}(F)$ we obtain (19). Hence, since $\left\langle x_{n}-x, L x_{n}\right\rangle_{V}=$ $\left\langle x_{n}, \theta_{n} F x_{n}\right\rangle_{V}-\left\langle x, \theta_{n} F x_{n}\right\rangle_{V}, x \in V=V_{0} \hookrightarrow \Gamma$ and $Y=\Gamma^{\prime}$, it follows that

$$
\begin{aligned}
& \limsup _{n \rightarrow \infty}\left\langle x_{n}-x, L x_{n}\right\rangle_{V} \\
& \quad=\limsup _{n \rightarrow \infty}\left\langle x_{n}, \theta_{n} F x_{n}\right\rangle+\liminf _{n \rightarrow \infty}\left(-\left\langle x, \theta_{n} F x_{n}\right\rangle\right) \\
& \quad=\limsup _{n \rightarrow \infty}\left\langle x_{n}, \theta_{n} F x_{n}\right\rangle-\langle x, F x\rangle \\
& \leq 0 .
\end{aligned}
$$

Consequently, by the Browder-Skrypnik property of $L \in \mathfrak{C}(B S), x_{n} \rightarrow x$ in the norm of $V$. By the demicontinuity of $L \in \mathfrak{C}(B S), L x_{n} \rightarrow L x$ weakly, and consequently; $L x=\kappa$. Thus, $F x=\kappa=L x$.

We consider the case when condition (iv) is satisfied. As in the case (i) one can prove that $\theta_{n} F x_{n}=L x_{n} \rightarrow F x=\kappa$ in the weak topology $\sigma\left(W, V_{0}\right)$, and by $V \in \mathfrak{N}(F)$ we obtain (19). Then

$$
\left\langle z, L x_{n}\right\rangle_{V}=\left\langle z, \theta_{n} F x_{n}\right\rangle_{V} \rightarrow\langle z, \kappa\rangle_{V}=\langle z, F x\rangle \quad\left(z \in V_{0}\right) .
$$

as $n \rightarrow \infty$. Further, we have

$$
\limsup _{n \rightarrow \infty}\left\langle x_{n}, L x_{n}\right\rangle_{V}=\limsup _{n \rightarrow \infty}\left\langle x_{n}, \theta_{n} F x_{n}\right\rangle \leq\langle x, F x\rangle=\langle x, \kappa\rangle_{V}
$$

By $L \in \mathfrak{C}(G M)$ it follows that $x \in D(L)$ and $L x=\kappa$. Thus, $F x=k=L x$

\section{Auxiliary lemmas}

We need else the following technical notions $[25-26]$ in the theory of Banach $L_{\infty}$ modules for some further proofs in this paper.

Let $B: \Omega \rightarrow 2^{\mathbb{R}^{m}}$ be a multifunction, for which there exists a sequence of measurable functions $\left\{x_{i}\right\}_{i \in \mathbf{N}}$ such that the closure hull of $\left\{x_{i}(s)\right\}_{i \in \mathbf{N}}$ coincides with $B(s)$ for a.a. $s \in \Omega$ and $B(s)$ is a symmetric absolutely convex compact subset of $\mathbb{R}^{m}$. Then $B$ is called (see [25 - 26]) an infra-semi-unit or $m$-unit of $X$ if $x \in X$ for every so-called measurable selector $x$ of $B$, i.e. measurable function $x$ such that $x(s) \in B(s)$ for a.a. $s \in \Omega$. In the scalar case $m=1$, any 1 -unit $B$ has the form $B(s)=[-b(s),+b(s)]$ where $b$ is a non-negative element in $X$. A subset $\mathfrak{N} \subset X$ is called (see [25-26]) $U$-bounded, if all elements of $\mathfrak{N}$ are measurable selectors for some fixed $m$-unit $B$ of $X$. In the scalar case $m=1$, this notion is equivalent to the notion of order-boundedness of $\mathfrak{N}$, i.e. to the existence of a non-negative function $b \in X$ such that $|x(s)| \leq b(s)$ for a.a. $s \in \Omega$ and for all $x \in \mathfrak{N}$. 
An analog of the space $\mathbb{L}_{\infty}$ is the Banach $L_{\infty}$-module $M_{B}$ of measurable functions $x$ with the norm

$$
\|x\|_{M_{B}}=\inf \{\lambda>0: x(s) \in \lambda B(s) \text { a.e. }\}<+\infty .
$$

A subset $\mathfrak{N}$ is $U$-bounded if and only if $\mathfrak{N}$ is bounded in the norm of $M_{B}$ for some $m$ unit of $X$. The associtate space $\left(M_{B}\right)^{\prime}$ (denoted by $L_{B^{\circ}}$ ) as an analog of the Lebesgue space $L_{1}$ is a regular space [26]. For example, in the scalar case $m=1$, when $B(s)=$ $[-b(s),+b(s)], L_{B^{\circ}}$ is just the Lebesgue space $L_{1}(b)$ with weight $b$.

Recall that an operator $F: X \rightarrow Y$ is called $U$-bounded on the whole space $X$, if $F(X)$ is $U$-bounded in $Y$.

Lemma 3. Let $V \hookrightarrow \hookrightarrow E, E \subset S\left(\Omega, \mathbb{R}^{m}\right)$ be a Banach $L_{\infty}$-module, $L \in \mathfrak{C}$, where $\mathfrak{C}$ is one of the classes $\mathfrak{C}(\mathcal{L}), \mathfrak{C}(B M)$ or $\mathfrak{C}(B S)$, and the superposition operator $F: E \rightarrow E^{\prime}$ be $U$-bounded on whole space $E$. Suppose that the following condition is satisfied:

(R1) There exists a superpositionally measurable function $H(\cdot, \cdot): \Omega \times \mathbb{R}^{m} \rightarrow[0, \infty)$ such that $(u, f(s, u)) \leq H(s, u)$, the superposition operator $H$ generated by $H(\cdot, \cdot)$ acts from $V$ into $L(\Omega, \mathbb{R})$, and $\langle x, L x\rangle_{V}-H x>0 \quad(\|x\| \geq r)$ for some $r>0$.

Then the equation $L x=F x$ has at least one solution $x^{*} \in T_{V}(r)$ for which $\left\|L x^{*}\right\| w \leq c$ where $c=\sup \left\{\|L z\|_{W}:\|z\|_{V} \leq r\right\}$.

Proof. Remember that $E \hookrightarrow S\left(\Omega, \mathbb{R}^{m}\right)$ by [27: Theorem 4]. First, we prove that $L-F \in \mathfrak{C}$. Since $F: E \rightarrow E^{\prime}$ is $U$-bounded on whole $E$, there exists an $m$-unit $B$ of $E^{\prime}$ such that $F(X) \subset M_{B} \hookrightarrow E^{\prime}, E \hookrightarrow\left(M_{B}\right)^{\prime}=L_{B^{\circ}}$, and $F: E \rightarrow M_{B}$ is a bounded operator on whole $E$ (see [27: Theorem 4] and [25: Theorem 5]). In the scalar case $m=1$, this 1-unit $B$ has the form $B(s)=[-b(s),+b(s)]$ where $b$ is a non-negative element in $E^{\prime}$ with $\operatorname{supp} b=\Omega$. Let $\left\|x_{n}-x\right\|_{E} \rightarrow 0$ as $n \rightarrow \infty$. Then $x_{n}$ converges in the norm of $L_{B^{\circ}}$ and, consequently, it has uniformly absolutely continuous norms as $L_{B^{\circ}}$ is regular [26] by the Vitali-Krasnoselskij type convergence theorem (see, e.g., [20] and [27: Theorem 8]). Hence, for $z \in L_{B^{\bullet}} \supset E$ we get

$$
\lim _{\operatorname{mes}(D) \rightarrow 0} \sup _{n} \int_{D}\left|\left(z, F x_{n}\right)\right| d s \leq \lim _{\operatorname{mes}(D) \rightarrow 0}\left\|P_{D} z\right\|_{L_{B} \circ} \sup _{n}\left\|F x_{n}\right\|_{M_{B}}=0 .
$$

By the Nemytskij theorem (see, e.g., $[5,20]) F x_{n} \rightarrow F x$ in the metric of $S\left(\Omega, \mathbb{R}^{m}\right)$, and hence it follows from the Vitali-Krasnoselskij convergence theorem in $L(\Omega, \mathbb{R})$ (see, e.g., [20] and [27: Theorem 8]) that $\left\langle F x_{n}, z\right\rangle \rightarrow\langle F x, z\rangle$ as $n \rightarrow \infty$, for every $z \in E$. Therefore, $F x_{n}$ converges in the weak topology $\sigma\left(E^{\prime}, E\right)$. This proves that $f: E \rightarrow$ $\left(E^{\prime}, \sigma\left(E^{\prime}, E\right)\right)$ is continuous.

We consider the case $\mathfrak{C}=\mathfrak{C}(\mathcal{L})$. Since $V \hookrightarrow \hookrightarrow E$ and $F: E \rightarrow E^{\prime}$ is $U$-bounded, boundedness of $F: V=V_{0} \rightarrow W=W_{0}$ follows and consequently, boundedness of $L-F: V \rightarrow W$, since $L \in \mathfrak{C}(\mathcal{L})$ is bounded. Further, since $V=V_{0} \hookrightarrow \hookrightarrow E$ and $V$ is a reflexive Banach space, from weak convergence in $V$ strong convergence in $E$ follows. Then the proved continuity of $F: E \rightarrow\left(E^{\prime}, \sigma\left(E^{\prime}, E\right)\right)$ implies weak continuity of $F: V \rightarrow W$, and consequently $L-F: V \rightarrow W$ is weakly continuous, since $L \in \mathfrak{C}(\mathcal{L})$ is weakly continuous. Thus, $L-F \in \mathfrak{C}(\mathcal{L})$. 
Next we consider the case $L \in \mathfrak{C}=\mathfrak{C}(B M)$. As in the first case we can prove that $L-F: V=V_{0} \rightarrow W=W_{0}$ is bounded and $F: V \rightarrow W$ is weakly continuous, in particular, $F: V \rightarrow W$ is weakly continuous on every finite-dimensional subspace of $V$. Since $L \in \mathfrak{C}(B M)$ is weakly continuous on every finite-dimensional subspace of $V$, $L-F$ has the same continuity property. It remains to prove that $L$ is an operator of type $(M)$. For this suppose that $x_{n} \rightarrow x$ weakly in $V,(L-F) x_{n} \rightarrow \kappa$ weakly in $W$ and $\lim \sup _{n \rightarrow \infty}\left\langle x_{n},(L-F) x_{n}\right\rangle_{V} \leq\langle x, \kappa\rangle_{V}$. By $V=V_{0} \hookrightarrow \hookrightarrow E, x_{n} \rightarrow x$ in the norm of $E$ and in the metric of $S\left(\Omega, \mathbb{R}^{m}\right)$. Consequently, $\left(x_{n}, F x_{n}\right) \rightarrow(x, F x)$ in $S(\Omega, \mathbb{R})$. Further, $F x_{n} \in M_{B}$ and $x_{n} \rightarrow x$ in $L_{B^{\circ}}$. We have

$$
\begin{aligned}
\lim _{\operatorname{mes}(D) \rightarrow 0} \sup _{n} \int_{D}\left|\left(x_{n}, F x_{n}\right)\right| d s & \leq \lim _{\operatorname{mes}(D) \rightarrow 0} \sup _{n}\left\|F x_{n}\right\|_{M_{B}}\left\|P_{D} x_{n}\right\|_{L_{B}} \\
& \leq c \lim _{\operatorname{mes}(D) \rightarrow 0} \sup _{n}\left\|P_{D^{\circ}} x_{n}\right\|_{L_{B^{\circ}}} \\
& =0
\end{aligned}
$$

where $c=\sup _{n}\left\|F x_{n}\right\|_{M_{B}}<\infty$, since $F: E \rightarrow M_{B}$ is bounded and $L_{B^{\circ}}$ is regular (see [26]). Hence it follows by the Vitali-Krasnoselskij theorem in $L(\Omega, \mathbb{R})$ (see, e.g., [20] or [27: Theorem 8]) that $\left(x_{n}, F_{x_{n}}\right) \rightarrow(x, F x)$ in $L(\Omega, \mathbb{R})$ and, in particular,

$$
\lim _{n \rightarrow \infty}\left\langle x_{n}, F x_{n}\right\rangle=\langle x, F x\rangle=\langle x, F x\rangle_{V}
$$

We have that $F x_{n} \rightarrow F x$ weakly in $W$ and, consequently, $L x_{n} \rightarrow \kappa+F x$ weakly. Thus,

$$
\begin{aligned}
\limsup _{n \rightarrow \infty}\left\langle x_{n}, L x_{n}\right\rangle_{V} & \leq \limsup _{n \rightarrow \infty}\left\langle x_{n},(L-F) x_{n}\right\rangle_{V}+\underset{n \rightarrow \infty}{\limsup _{n}}\left\langle x_{n}, F x_{n}\right\rangle \\
& \leq\langle x, \kappa\rangle_{V}+\langle x, F x\rangle_{V} \\
& =\langle x, \kappa+F x\rangle_{V} .
\end{aligned}
$$

Consequently, $L x=\kappa+F x$, since $L \in \mathfrak{C}(B M)$ is an operator of type $(M)$. This completes the proof of $L-F \in \mathfrak{C}(B M)$.

At last we consider now the case that $L \in \mathfrak{C}(B S)$. As in the preceding case one can prove that $F: V=V_{0} \rightarrow W=W_{0}$ is bounded and weakly continuous, in particular, demicontinuous. Since $L \in \mathfrak{C}(B S)$ is bounded and demicontinuous, $L-F$ has also these properties. It remains to prove the Browder-Skrypnik property of $L-F \in \mathfrak{C}(B S)$. For this suppose that $x_{n} \rightarrow x$ weakly and $\lim _{n \rightarrow \infty}\left(x_{n}-x,(L-F) x_{n}\right)_{V} \leq 0$. Arialogously as in the proof of (24) one can prove that

$$
\lim _{n \rightarrow \infty}\left\langle x_{n}-x, F x_{n}\right\rangle=0 .
$$

Hence it follows that

$$
\begin{aligned}
\lim _{n \rightarrow \infty}\left\langle x_{n}-x, L x_{n}\right\rangle_{v} & =\lim _{n \rightarrow \infty}\left\langle x_{n}-x,(L-F) x_{n}\right\rangle_{V}+\lim _{n \rightarrow \infty}\left\langle x_{n}-x, F x_{n}\right\rangle_{V} \\
& \leq 0+0 \\
& =0 .
\end{aligned}
$$


By the Browder-Skrypnik property of $L \in \mathfrak{C}(B S)$ it follows that $x_{n} \rightarrow x$ in the norm of $V$. This proves that $L-F$ has the Browder-Skrypnik property and that $L-F \in \mathfrak{C}(B S)$.

Thus we proved that $L-F \in \mathfrak{C}$. By condition (Rl) of Lemma we have

$$
(u, f(s, u)) \leq H(s, u) \geq 0
$$

and

$$
\langle x,(L-F) x\rangle_{V}=\langle x, L x\rangle_{V}-\langle x, F x\rangle \geq\langle x, L x\rangle_{V}-H x>0 \quad\left(\|x\|_{V} \geq r>0\right) .
$$

Then by well-known existence theorems for operators of the class $\mathfrak{C}$ (see, e.g., $[11,21$, $29,31-32,38])$ it follows that $L x=F x$ has at least one solution $x^{*} \in T_{V}(r)$

Lemma 4. Let $V \hookrightarrow \hookrightarrow E, E \subset S\left(\Omega, \mathbb{R}^{m}\right)$ be a Banach $L_{\infty}$-module, $L \in \mathfrak{C}(G M)$ and the superposition operator $F: E \rightarrow E^{\prime}$ be $U$-bounded on whole space $E$. Suppose that the following condition is satisfied:

(R2) There exist a function $y \in V_{0}$, a superpositionally measurable function $H(\cdot, \cdot)$ : $\Omega \times \mathbb{R}^{m} \rightarrow[0, \infty)$ and a number $r>0$ such that $(u-y(s), f(s, u)) \leq H(s, u)$, the superposition operator $H: V \rightarrow L(\Omega, \mathbb{R})$ generated by $H(\cdot, \cdot)$ is bounded on bounded sets, $\langle x-y, L x\rangle_{V}-H_{x}>0\left(\|x\|_{V} \geq r>0\right)$, and $\|L x\|_{W} \leq c\left(c_{1}, c_{2}\right)<$ $\infty$ where $\|x\| \leq c_{1}<\infty$ and $\langle x-y, L x\rangle_{V} \leq c_{2}<\infty$.

Then the equation $L x=F x$ has at least one solution $x^{*}$ for which $\left\|L x^{*}\right\| W \leq c^{\prime}$ where $c^{\prime}=c^{\prime}\left(r, \sup _{\|z\| v} \leq r\|H z\|_{L}\right)<\infty$.

Proof. Analogously as in the proof of Lemma 3 in the case $L \in \mathfrak{C}(B M)$ one can prove that $L-F \in \mathfrak{C}(G M)$ and that $F$ is bounded in $W$ on every bounded in $V$ subset. Further, we prove that $L-F$ satisfies also condition (R2) for same $y$ and $H(s, u)$ and for some function $c^{\prime}\left(c_{1}, c_{2}\right)$. First we have

$$
\begin{aligned}
(u-y(s), f(s, u)) & \leq H(s, u) \geq 0 \\
\langle x-y,(L-F) x\rangle_{V} & =\langle x-y, L x\rangle_{V}-\langle x-y, F x\rangle_{V} \\
& \geq\langle x-y, L x\rangle_{V}-H x \\
& >0 \quad\left(\|x\|_{V} \geq r>0\right) .
\end{aligned}
$$

Now let $\|x\|_{V} \leq c_{1}<\infty$ and $\langle x-y,(L-F) x\rangle_{V} \leq c_{2}<\infty$. Then $x \in D(L)$ (since $L x=(L-F) x+F x)$ and

$$
\begin{aligned}
\langle x-y, L x\rangle_{V} & =\langle x-y,(L-F) x\rangle_{V}+\langle x-y, F x\rangle \\
& \leq c_{2}+\left(\|x\|_{v}+\|y\|_{V}\right) \sup _{\|z\|_{v} \leq c_{1}}\|f z\|_{W} \\
& =c_{2}^{\prime} \\
& <\infty
\end{aligned}
$$

Consequently, by condition (R2) for $L$ we have $\|L x\|_{w} \leq c\left(c_{1}, c_{2}^{\prime}\right)<\infty$ where $\|F x\| w \leq$ $d\left(c_{1}\right)=\sup _{\|z\|_{v} \leq c_{1}}\|f z\|_{W}<\infty$. Hence

$$
\|(L-F) x\|_{W} \leq c^{\prime}\left(c_{1}, c_{2}\right)=c\left(c_{1}, c_{2}^{\prime}\right)+d\left(c_{1}\right)
$$


follows where $\|x\|_{V} \leq c_{1}<\infty$ and $\langle x-y,(L-F) x\rangle_{V} \leq c_{2}<\infty$. By $(L-F) \in \mathfrak{C}(G M)$ and (26) - (27), from known existence theorems (see, e.g., $[13,17])$ it follows that the equation $L x=F x$ has at least one solution $x^{*} \in T_{V}(r)$. The estimation of the norm $\left\|L x^{*}\right\|_{W}$ follows from condition (R2) and the relations $\left\langle x^{*}-y, L x^{*}\right\rangle=\left\langle x^{*}-y, F x^{*}\right\rangle_{V} \leq$ $\left\|H x^{*}\right\|_{L}$

\section{Main existence theorem in the usual weak sense}

We state now the general result on the existence of solutions in $V$ for the problem $L x \doteq F x$ via the abstract notion $\left(V, V_{0} ; W, W_{0}\right) \in \mathfrak{N}(L, F)$. We draw attention of the reader to the fact that verifiable sufficient conditions for $\left(V, V_{0} ; W, W_{0}\right) \in \mathfrak{N}(L, F)$ were given before in Proposition 3 (Section 5) together with Proposition 2 (Section 5) and Proposition 1 with Corollaries 1 - 2 (Section 4).

Theorem 1. Let $\left(V, V_{0} ; W, W_{0}\right)$ be a complementary system in the sense of Section 3 , and let $E, Y, \Gamma \subset S\left(\Omega, \mathbb{R}^{m}\right)$ be Banach $L_{\infty}$-modules such that $V \hookrightarrow \hookrightarrow E, \Gamma^{\prime}=Y$ and $V_{0} \hookrightarrow \Gamma$. Further, suppose that one of the following conditions is satisfied:

(i) $\left(V, V_{0} ; W, W_{0}\right) \in \mathfrak{N}(L, F)$, and condition (R1) in Lemma 3 for $L \in \mathbb{C}$ is satisfied where $\mathfrak{C}$ is one of the classes $\mathfrak{C}(\mathcal{L}), \mathfrak{C}(B S), \mathfrak{C}(B M)$.

(ii) $\left(V, V_{0} ; W, W_{0}\right) \in \mathfrak{N}(L, F)$, and condition (R2) in Lemma 4 for $L \in \mathfrak{C}(G M)$ is satisfied.

Then the equation $L x=F x$ has at least one solution in $V$.

Proof. Fix an $m$-unit $B$ of $E^{\prime}$ such that the linear hull of $B(s)$ coincides with $\mathbb{R}^{m}$ for a.a. $s \in \Omega$ (see Sections 1 and 6, and [25: Theorem 5]; in the scalar case $m=1$, this 1-unit $B$ has the form $B(s)=[-b(s),+b(s)]$ where $b$ is a non-negative element in $E^{\prime}$ with $\operatorname{supp} b=\Omega)$. We define the Carathéodory function $\varphi_{B}(s, u)=\inf \{\lambda>0: u \in \lambda B(s)\}$, and then $\varphi_{B}(s, \cdot)$ is a norm in $\mathbb{R}^{m}$ for a.a. $s \in \Omega$. Put

$$
f_{n}(s, u)=\min \left\{1, n\left(\varphi_{B}(s, f(s, u))\right)^{-1}\right\} f(s, u) .
$$

Evidently, for each $n \in \mathbb{N}, f_{n}$ [the so-called relaxation of $f$ ] is a Carathéodory function and it defines the superposition operator $F_{n}: E \rightarrow E^{\prime}$, which is $U$-bounded on whole space $E$, since $F_{n}(X)$ is contained in the ball of $M_{B}$ with radius $n$. Further, for the sequence of scalar functions

$$
\theta_{n}(s, u)=\min \left\{1, n\left(\varphi_{B}(s, f(s, u))\right)^{-1}\right\}
$$

we have $\theta_{n} \rightarrow 1$ a.e. and, therefore, $f_{n} \rightarrow f$ a.e. In the end, for each function $f_{n}$ condition (R1) in Lemma 3 or condition (R2) in Lemma 4 holds. By Lemmas 3 and 4 the equation $L x=F_{n} x_{n}$ has at least one solution $x_{n} \in T_{V}(r)$ for which $\left\|L x_{n}\right\|_{w}$. $c<\infty$ for some numbers $r, c>0$ not depending on $n$. Obviously, $L x_{n}=\theta_{n} F x_{n}$ where $\theta_{n}(\cdot)=\theta_{n}\left(s, x_{n}(\cdot)\right) \in \Xi$ and $\theta \rightarrow 1$ in $S(\Omega, \mathbb{R})$.

By $V \hookrightarrow \hookrightarrow E \hookrightarrow S\left(\Omega, \mathbb{R}^{m}\right)$ (from our assumptions and [27: Theorem 4]) and by the Alaoglu-Bourbaki theorem [12] hence the existence of a subsequence $\left\{n_{k}\right\}$ follows such that, for some $x^{*} \in T_{V}(r), x_{n_{k}} \rightarrow x^{*}$ in the weak topology $\sigma\left(V, W_{0}\right)$, in norm of $E$ and in the metric of $S\left(\Omega, \mathbb{R}^{m}\right)$, and, for some $\kappa \in T_{W}(r), L x_{n_{k}} \rightarrow \kappa$ in the weak topology $\sigma\left(W, V_{0}\right)$. By $\left(V, V_{0} ; W, W_{0}\right) \in \mathfrak{N}(L, F)$ hence it follows that $L x^{*}=\kappa=F x^{*}$, i.e. the equation $L x=F x$ has a solution in $V$ 


\section{Main existence theorem in the $\Gamma$-weakened sense}

8.1 Solvability in the $\Gamma$-weakened sense. Let $\left(V, V_{0} ; W, W_{0}\right)$ be a complementary system in the sense of Section 3. Recall that the equation $L x=F x$ is solvable in the $\Gamma$-weakened sense (here $\Gamma$ is some fixed Banach $L_{\infty}$-module in $S\left(\Omega, \mathbb{R}^{m}\right)$ ) if there exists $x$ * such that

$$
\begin{aligned}
\left\langle y, L x_{*}\right\rangle_{V} & =\left\langle y, F x_{*}\right\rangle \quad(y \in V \cap \Gamma) \\
\left\langle x_{*}, L x_{*}\right\rangle_{V} & =\left\langle x_{*}, F x_{*}\right\rangle .
\end{aligned}
$$

Remark that the solvability in the $L_{\infty}$-weakened sense is considered, e.g., in [6 - 10,15 $-16,18,34$ ] (see also the literature cited therein). In this section we get the general Theorem 2 .on the existence in the $\Gamma$-weakened sense by means of the method presented in Sections 4 - 7 that refines results of these mathematicians for the case when $\Gamma=L_{\infty}$ and $m=1$. From this Theorem it follows that the so-called "sign condition" in the above mentioned articles is not sufficient for the solvability of the equation $L_{x}=F_{x}$ in the vector case $m \neq 1$.

We state now the general result on the existence of solutions in the $\Gamma$-weakened sense in $V$ for the problem $L x=F x$ via the following abstract notion $\left(V, V_{0} ; W, W_{0}\right) \in$ $\mathfrak{N}(L, F, \Gamma)$. We draw attention of the reader to the fact that verifiable sufficient conditions for the inclusion $\left(V, V_{0} ; W, W_{0}\right) \in \mathfrak{N}(L, F, \Gamma)$ will be given in Proposition 5 (Subsection 8.2) together with Proposition 4 (Subsection 8.2), the (mentioned in the first part of Subsection 8.2] sufficient conditions for the inclusion $\left(V, V_{0} ; W, W_{0}\right) \in \operatorname{Tr}\left(V_{0}, \Gamma\right)$, and Proposition 1 and its Corollaries 1 - 2 (Section 4).

Let $\mathfrak{C}$ be a fixed class from $\mathfrak{C}(B M)$ or $\mathfrak{C}(G M)$ (see Section 5). Following Section 6 , we recall that $\left(V, V_{0} ; W, W_{0}\right) \in \mathfrak{N}(L, F, \Gamma)$ if for any sequences $\left\{x_{n}\right\} \subset D(L)$ and $\left\{\theta_{n}\right\} \subset \Xi$ such that

$$
\begin{aligned}
& L x_{n}=\theta_{n} F x_{n} \\
& x_{n} \rightarrow x_{*} \text { in the metric of } S\left(\Omega, \mathbb{R}^{m}\right) \\
& x_{n} \rightarrow x^{*} \text { in the weak topology } \sigma\left(V, W_{0}\right) \\
& \theta_{n} \rightarrow 1 \text { in } S(\Omega, \mathbb{R}) \\
& L x_{n} \rightarrow \kappa \text { in the weak topology } \sigma\left(W, V_{0}\right)
\end{aligned}
$$

it follows that $L x_{*}=\kappa$ and relations (29) - (30) hold. Remember that, as before, $\Xi$ is the set of all measurable scalar functions $\theta$ such that $0 \leq \theta(s) \leq 1$.

Theorem 2. Let $\left(V, V_{0} ; W, W_{0}\right)$ be a complementary system in the sense of Section 3 , and $E, Y, \Gamma \subset S\left(\Omega, \mathbb{R}^{m}\right)$ be Banach $L_{\infty}$-modules such that $V \hookrightarrow \hookrightarrow E$ and $\Gamma^{\prime}=Y$ is regular (in particular, $\Gamma=\mathbb{L}_{\infty}$ ). Further, suppose that one of the following conditions is satisfied:

(i) $\left(V, V_{0} ; W, W_{0}\right) \in \mathfrak{N}(L, F, \Gamma)$ and condition (R1) in Lemma 3 is satisfied for $L \in \mathfrak{C}(B M)$.

(ii) $\left(V, V_{0} ; W, W_{0}\right) \in \mathfrak{N}(L, F, \Gamma)$ and condition (R2) in Lemma 4 is satisfied for $L \in \mathfrak{C}(G M)$.

Then the equation $L x=F x$ has at least one solution $x * \in V$ in the $\Gamma$-weakened sense. 
Proof. Let $f_{n}$ be the functions defined in (28) and $\theta_{n}$ be the functions in the proof of Theorem 1. As proved there, each equation $L x=F_{n} x$ has at least one solution $x_{n}$ such that $x_{n} \in T_{V}(r)$ and $\left\|L x_{n}\right\|_{W} \leq c<\infty$ for some $r, c>0$ not depending on $n$. Obviously, $L x_{n}=\theta_{n} F x_{n}$ where $\theta_{n}(\cdot)=\theta_{n}\left(\cdot, x_{n}(\cdot)\right) \rightarrow 1$ in $S(\Omega, \mathbb{R})$. By $V \hookrightarrow \hookrightarrow E \hookrightarrow S\left(\Omega, \mathbb{R}^{m}\right)$ (from our assumptions and [27: Theorem 4]) and the Alaoglu-Bourbaki theorem [12] hence there follows the existence of a subsequence $\left\{n_{k}\right\}$ such that, for some $x^{*} \in T_{V}(r)$, $x_{n_{k}} \rightarrow x *$ in the weak topology $\sigma\left(V, W_{0}\right)$, in the norm of $E$ and in the metric of $S\left(\Omega, \mathbb{R}^{m}\right)$, and, for some $\kappa \in T_{W}(c), L x_{n_{k}} \rightarrow \kappa$ in the weak topology $\sigma\left(W, V_{0}\right)$. By $\left(V, V_{0} ; W, W_{0}\right) \in \mathfrak{N}(L, F, \Gamma)$ hence $L x_{*}=\kappa$ and relations (29) - (30) follow

8.2. Verifiable sufficient conditions for $\left(V, V_{0} ; W, W_{0}\right) \in \mathfrak{N}(L, F, \Gamma)$. The verification of the inclusion $\left(V, V_{0} ; W, W_{0}\right) \in \mathfrak{N}(L, F, \Gamma)$ is connected with the inclusion $(Y, \Gamma) \in \mathfrak{Z} v(L, F, \mathfrak{F})$ and with "good" properties of the spaces $V$ and $V_{0}$. We recall that $\left(V, V_{0} ; W, W_{0}\right) \in \operatorname{Tr}\left(V_{0}, \Gamma\right)$ if the following two conditions are satisfied:

(T1) (Brezis-Browder-Gossez condition). From that $\kappa \in W, z \in \Gamma^{\prime},\langle y, \kappa\rangle_{V}=$ $\langle y, z\rangle \quad\left(y \in V_{0} \cap \Gamma\right), x \in V,(x(s), z(s)) \leq h(s)$ for a.a. $s \in \Omega$ and $h \in L(\Omega)$ it follows that $\langle x, \kappa\rangle_{V}=\langle x, z\rangle$.

(T2) For each $y \in V \cap \Gamma$ there exists $y_{j} \in V_{0} \cap \Gamma$ such that $y_{j} \rightarrow y$ in the weak topology $\sigma(V, W)$ and in the metric of $S\left(\Omega, \mathbb{R}^{m}\right)$, and $\sup _{j \in \mathbb{N}}\|y j\|_{\Gamma}<\infty$.

The verification of the inclusion $\left(V, V_{0} ; W, W_{0}\right) \in \operatorname{Tr}\left(V_{0}, \Gamma\right)$ in the non-trivial case $V_{0} \cap$ $\Gamma \neq V_{0}$ is connected with many considerable difficulties. At the present from results of H. Brezis and F.E. Browder [7 - 8], J.-P. Gossez [15 - 16], A. Benkirane and J.-P. Gossez [6] it follows that $\left(V, V_{0} ; W, W_{0}\right) \in \operatorname{Tr}\left(V_{0}, \Gamma\right)$ if one of the following conditions is satisfied:

(i) $V=V_{0}=\dot{W}_{p}^{k}(\Omega)$ and $\Gamma=L_{\infty}(\Omega)$ (see $\left.[7-8]\right)$.

(ii) $V=V_{0}=W_{p}^{k}(\Omega), \Gamma=L_{\infty}(\Omega)$ and $\Omega$ satisfies the cone condition and the segment condition (see $[7-8]$ ).

(iii) $V=\dot{W}^{1} L_{M}(\Omega), V_{0}=\dot{W}^{1} L_{M}^{\circ}(\Omega)$ and $\Gamma=L_{\infty}(\Omega)$ where $\Omega$ satisfies the segment condition (see $[15-16]$ ).

(iv) $V=W^{1} L_{M}(\Omega), V_{0}=W^{1} L_{M}^{\circ}(\Omega)$ and $\Gamma=L_{\infty}(\Omega)$ where $\Omega$ satisfies the cone condition and the segment condition (see [15 - 16]).

(v) $V=\dot{W}^{k} L_{M}(\Omega), V_{0}=\dot{\circ}^{k} L_{M}^{\circ}(\Omega)$ and $\Gamma=L_{\infty}(\Omega)$ where $k \geq 2$ and $\Omega$ satisfies the segment condition, and both Young functions $M$ and $M^{*}$, satisfy the $\Delta_{2}$-condition (see [6]).

As an analog to the inclusion $V \in \mathfrak{N}(F)$ in Section 6 we recall that $V \in \mathfrak{N}^{\prime}(F)$ if for any sequences $\left\{x_{n}\right\}$ and $\left\{\theta_{n}\right\}$ such that $x_{n} \rightarrow x$ in the weak topology $\sigma\left(V, W_{0}\right)$ and in the metric of $S\left(\Omega, \mathbb{R}^{m}\right), \theta_{n} \rightarrow 1$ in $S(\Omega, \mathbb{R})$ and $\left|\left\langle x_{n}, \theta_{n} F x_{n}\right\rangle\right|<\infty$ it follows that

$$
\limsup _{n \rightarrow \infty}\left\langle x_{n}, \theta_{n} F x_{n}\right\rangle \leq\langle x, F x\rangle<\infty
$$

and $(x(s), F x(s)) \leq h(s)$ holds for a.a. $s \in \Omega$ where $h$ is some function of $L(\Omega, \mathbb{R})$. 
Proposition 4. Let $(u, f(s, u)) \leq B(s, u) \geq 0$ with $B(\cdot, \cdot)$ a Carathéodory function generating the superposition operator $B: Z * \rightarrow L(\Omega, \mathbb{R})$ where $V \hookrightarrow \hookrightarrow Z$ * and $Z * \subset$ $S\left(\Omega, \mathbb{R}^{m}\right)$ is a Banach $L_{\infty}$-module. Then $V \in \mathfrak{N}^{\prime}(F)$.

Proof. By Proposition 2 we have $V \in \mathfrak{N}(F)$. Since $x \in V \hookrightarrow Z_{*}$, we have $(x(s), F x(s)) \leq h(s), h(s)=B x(s) \geq 0$ and $h \in L(\Omega, \mathbb{R})$. This completes the proof of $V \in \mathfrak{N}^{\prime}(F)$

Proposition 5. Let $\Gamma^{\prime}=Y$ be a regular Banach $L_{\infty}$-module (in particular, $\Gamma=$ $\left.\mathbb{L}_{\infty}\right),(Y, \Gamma) \in Z_{v}(L, F, \mathcal{F}), V \in \mathfrak{N}^{\prime}(F),\left(V, V_{0} ; W, W_{0}\right) \in \operatorname{Tr}\left(V_{0}, \Gamma\right)$, and $L \in \mathfrak{C}$ where $\mathfrak{C}$ is one of the classes $\mathfrak{C}(B M)$ or $\mathfrak{C}(G M)$. Then $\left(V, V_{0} ; W, W_{0}\right) \in \mathfrak{N}(L, F, \Gamma)$.

Proof. Let $x_{n}, \theta_{n}, \kappa, x_{*}$ be functions in the definition of $\left(V, V_{0} ; W, W_{0}\right) \in \mathfrak{N}(L, F, \Gamma)$. Then by $(Y, \Gamma) \in 3 v(L, F, \mathcal{F})$ and by the results of the proof of Proposition 2 (see (21)) we have $\lim _{n \rightarrow \infty}\left(y, \theta_{n} F x_{n}\right\rangle=\left\langle y, F x_{*}\right\rangle(y \in \Gamma)$ and, consequently,

$$
\langle y, \kappa\rangle_{V}=\lim _{n \rightarrow \infty}\left\langle y, L x_{n}\right\rangle_{V}=\lim _{n \rightarrow \infty}\left\langle y, \theta_{n} F x_{n}\right\rangle=\left\langle y, F x_{*}\right\rangle \quad\left(y \in V_{0} \cap \Gamma\right) .
$$

By $V \in \mathfrak{N}^{\prime}(F)$ we obtain

$$
\limsup _{n \rightarrow \infty}\left\langle x_{n}, L x_{n}\right\rangle_{V}=\underset{n \rightarrow \infty}{\limsup }\left\langle x_{n}, \theta_{n} F x_{n}\right\rangle \leq\left\langle x_{*}, F x_{*}\right\rangle<\infty
$$

and $\left(x_{*}(s), F x_{*}(s)\right) \leq h(s)$ for some $h \in L(\Omega, \mathbb{R})$. By (31) and condition (T1) in the definition of $\left(V, V_{0} ; W, W_{0}\right) \in \operatorname{Tr}\left(V_{0}, \Gamma\right)$ hence

$$
\left\langle x_{*}, \kappa\right\rangle_{V}=\left\langle x_{*}, F x_{*}\right\rangle
$$

follows. Consequently, by (32) we have

$$
\limsup _{n \rightarrow \infty}\left\langle x_{n}, L x_{n}\right\rangle_{V} \leq\left\langle x_{*}, \kappa\right\rangle_{V}
$$

Since $\mathfrak{C}=\mathfrak{C}(B M)$ or $\mathfrak{C}=\mathfrak{C}(G M), L x_{*}=\kappa$ follows.

Now by condition (T2) in the definition of $\left(V, V_{0} ; W, W_{0}\right) \in \operatorname{Tr}\left(V_{0}, \Gamma\right)$ there exists for any $y \in V \cap \Gamma$ a sequence $\left\{y_{j}\right\} \subset V_{0} \cap \Gamma$ such that $y_{j} \rightarrow y$ in the weak topology $\sigma(V, W)$ and in the metric of $S\left(\Omega, \mathbb{R}^{m}\right)$, and $\sup _{j}\left\|y_{j}\right\|<\infty$. Then, by the regularity of $\Gamma^{\prime}=Y$ we have

$$
\lim _{\operatorname{mes}(D) \rightarrow 0} \sup _{j} \int_{D}\left|\left(y_{j}(s), F x_{*}(s)\right)\right| d s \leq \sup _{j}\left\|y_{j}\right\|_{\Gamma} \lim _{\operatorname{mes}(D) \rightarrow 0}\left\|P_{D} F x_{*}\right\| Y=0 .
$$

Consequently, by the Vitali-Krasnoselskij convergence theorem (see, e.g., [20] or [27: Theorem 8]), $\left(y_{j}(\cdot), F x_{*}(\cdot)\right) \rightarrow\left(y(\cdot), F x_{*}(\cdot)\right)$ in $L(\Omega, \mathbb{R})$ and, in particular, $\lim _{j \rightarrow \infty}\left\langle y_{j}\right.$, $\left.F x_{*}\right\rangle=\left\langle y, F x_{*}\right\rangle$. From $(31)$ hence it follows that

$$
\langle y, \kappa\rangle_{V}=\lim _{j \rightarrow \infty}\left\langle y_{j}, \kappa\right\rangle_{V}=\lim _{j \rightarrow \infty}\left\langle y_{j}, F x_{*}\right\rangle=\left\langle y, F x_{*}\right\rangle \quad(y \in V \cap \Gamma) .
$$

From the proved relations $L \dot{x}_{*}=\kappa$ and (33) - (34) it follows that $x_{*} \in D(L)$ and relations (29) - (30) hold 


\section{Applications to boundary value problems in nonlinear mechanics}

In this Section by studying two concrete examples we explain the scheme of applications of Theorems 1 and 2 to some "perturbed" applied boundary value problems in Nonlinear Mechanics (mechanics of visco-plastic media, mechanics of elastic media, etc.) of the type $L x=F x$ where $x(s)$ is the "state" of the system, and the perturbation term $F x(s)=f[s, x(s)]$ is the "force" acting on the system and depending on the "state".

First we consider the "perturbed" boundary value problem finding the elastic-plastic torsion of strengthened bars

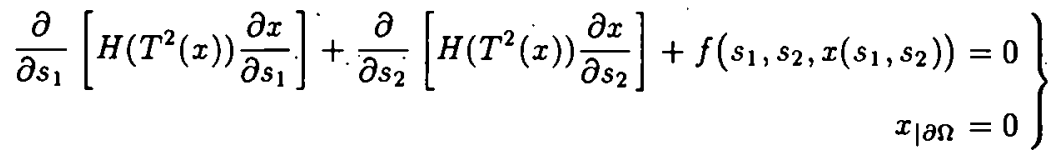

where $\Omega$ is a bounded domain of $\mathbb{R}^{2}, T^{2}(x)=\left(\frac{\partial x}{\partial s_{1}}\right)^{2}+\left(\frac{\partial x}{\partial s_{2}}\right)^{2}$, and $H(\alpha)$ characterizes material properties of a bar. Following [31], suppose that $H(\alpha)$ is continuous for $\alpha>0$, $a_{0}+a_{1} \alpha^{\frac{p}{2}-1} \leq H(\alpha) \leq A_{0}+A_{1} \alpha^{\frac{p}{2}-1}\left(\alpha \geq 0, p>1, a_{0} \geq 0, a_{1}>0\right)$ and $A_{0}=0$ for $1<p<2$. Then by [31] the system (35) is equivalent to the operator equation $L x=F x$ in $\stackrel{\circ}{W}_{p}^{1}(\Omega)$ where $L: \stackrel{\circ}{W}_{p}^{1}(\Omega) \rightarrow W_{p^{\prime}}^{-1}(\Omega)$ is an operator of the class $\mathfrak{C}(B S)$ and

$$
\langle L x, x\rangle=\iint_{\Omega} H\left(T^{2}(x)\right) T^{2}(x) d s d t \rightarrow+\infty \quad\left(\|x\|_{\dot{W}_{p}^{1}(\Omega)} \rightarrow+\infty\right) .
$$

From Theorem 1 and embedding theorems of S. I. Sobolev and S. I. Pokhozaev - N. S. Trudinger mentioned in Section 2, we have the following

Corollary 3. Let $Z=\mathbb{L}_{\frac{2 p}{2-p}}$ for $p<2, Z=L_{\infty}$ for $p>2, Z=L_{\varphi}^{\circ}$ for $p=2$ where $\varphi(s, u)=\exp u^{2}-1$. Suppose the following:

(i) $(u, f(s, u)) \leq b(s)$ for a.a. $s \in \Omega$ where $b \in L_{1}(\Omega, \mathbb{R})$.

(ii) $Z=\Gamma$, and the superposition operator $f$ acts from $\Gamma$ into $\Gamma^{\prime}$.

Then the boundary value problem (35) has at least one solution $x$ of $\dot{W}_{p}^{1}(\Omega, \mathbb{R})$.

Now we are going to consider the "perturbed" three-dimensional problem finding the elastic-plastic equilibrium of bodies in Plastic Deformation Theory, i.e.

$$
\left.\begin{array}{rl}
\sum_{j=1}^{3} \frac{\partial \sigma_{i j}}{\partial s_{j}}+\rho f_{j}(s, x(s)) & =0 \quad(i=1,2,3) \\
x_{\mid \partial \Omega} & =0
\end{array}\right\}
$$

where $\Omega \subset \mathbb{R}^{3}$ is a considered body, $\rho$ is its density, $x=\left(x_{1}, x_{2}, x_{3}\right)$ is the displacement vector, $f(s, x(s))=\left(f_{j}(s, x(s)) \quad(i=1,2,3)\right.$ is the "perturbed" mass force depending on a displacement vector, $\sigma_{i j}$ are the components of the stress tensor defined by means 
of the deformation tensor $\varepsilon_{i j}$ as $\sigma_{i j}(x)=\frac{e(x)}{2 k} \delta_{i j}+2 g(\Gamma(x))\left\{\varepsilon_{i j}(x)-\varepsilon(x) \delta_{i j}\right\}$ where $k$ is the volume compression coefficient, $\delta_{i j}$ are the Kronecker symbols,

$$
\begin{aligned}
\varepsilon_{i j}(x) & =\varepsilon_{j i}(x)=\frac{1}{2}\left(\frac{\partial x_{i}}{\partial s_{j}}+\frac{\partial x_{j}}{\partial s_{i}}\right), \quad \Gamma(x)=\left(2 \sum_{i, j} e_{i, j}(x)\right)^{\frac{1}{2}} \\
\varepsilon(x) & =\sum_{i, j} \varepsilon_{i j} \delta_{i j}=\sum_{i} \varepsilon_{i i}(x), \quad e_{i j}(x)=\varepsilon_{i j}(x)-\frac{1}{3} \varepsilon(x) \delta_{i j}
\end{aligned}
$$

$g$ is some function (so-called "plastic module") characterizing properties of a given material. Following [31], we suppose that $g$ is continuous on $\alpha>0$, and that $a_{0}+$ $a_{1} \alpha^{p-2} \leq g(\alpha) \leq A_{0}+A_{1} \alpha^{p-2}\left(\alpha \geq 0, p>1, a_{0} \geq 0, a_{1}>0\right)$ and $A_{0}=0$ for $1<p<2$. Then by [31] the system (36) is equivalent to the operator equation $L x=\rho F x$ in $W_{p}^{1}(\Omega)$ where $L: \dot{W}_{p}^{1}(\Omega) \rightarrow W_{p^{\prime}}^{-1}(\Omega)$ is an operator of the class $\mathfrak{C}(B S)$ and

$$
\langle L x, x\rangle \geq \int_{\Omega}\left\{\frac{\varepsilon^{2}(x)}{2 k}+g(\Gamma(x)) \Gamma^{2}(x)\right\} d s \rightarrow+\infty \quad\left(\|x\|_{W_{p}^{1}(\Omega)} \rightarrow+\infty\right) .
$$

From Theorem 1 and embedding theorems of S. I. Sobolev and S. I. Pokhozaev - N. S. Trudinger mentioned in Section 2, it follows

Corollary 4. Let $Z=\mathbb{L}_{\frac{3 p}{3-p}}$ for $p<3, Z=L_{\infty}$ for $p>3, Z=\mathbb{L}_{\varphi}^{\circ}$ for $p=3$ where $\varphi(s, u)=\exp \left(\|u\|^{\frac{3}{2}}\right)-1$. Suppose that condition (i) in Corollary 3 is satisfied and that for some Banach $L_{\infty}$-modules $X, \Gamma \subset S\left(\Omega, \mathbb{R}^{3}\right)$ the following condition is satisfied:

\section{(iii) Either}

(a) $Z \subset X$ and $Z \subset \Gamma$ with $\Gamma$ regular, and the superposition operator $\rho F$ acts from $X$ into $\Gamma^{\prime}$

or

(b) $Z \subset X$ and $Z \subset \Gamma$, and $\rho F: X \rightarrow \Gamma^{\prime}$ maps every bounded subset into a $\Gamma$-weakly absolutely bounded set

or else

(c) $Z \subset \Gamma$, the embedding $Z \subset X$ is absolutely bounded and $\rho F$ acts from $X$ into $\Gamma^{\prime}$.

Then the boundary value problem (36) has at least one solution $x$ * of $\dot{W}_{p}^{1}\left(\Omega, \mathbb{R}^{3}\right)$.

We remark that from the conditions of Corollaries 3 or 4 it follows that $L-F$ is bounded and demicontinuous, but it does not follow that $L-F$ has the BrowderSkrypnik property, and therefore, generally speaking, $(L-F) \notin \mathfrak{C}(B S)$, and so the general existence theorem of I. V. Skrypnik [31] is not applicable.

In conclusion of this section we remark that Corollaries 3 and 4 remain true if we replace in (35) - (36) $y_{0}+f(\cdot, \cdot)$ by $f(\cdot, \cdot)$ where $y_{0}$ is a fixed element of $W_{p^{\prime}}^{-1}(\Omega)$ ), since we can apply Theorem 1 for $\left(L x-y_{0}\right)=F x$ instead of $L x=F x$. 
Acknowledgement. The author acknowledges with many thanks the generous financial supports and research grants he has received from the All-Polish Research Foundation (KBN, Warsaw), namely the grant No 0705/P3/94/06 "Multivalued infrasemi-units and Banach $L_{\infty}$-modules" and the financial supports for his individual research projects "Nonlinear operator equations, multivalued operators, and evolutionary and non-evolutionary inclusions" (from 1995).

The results of the present paper and related results have been presented from 1992 up to the present in various research seminars at the Szczecin University (Poland), the University of Düsseldorf, the Free University of Berlin, and at the Fifth Vietnamese Mathematical Conference (1997). The author likes to acknowledge members of these seminars for their discussions and valuable remarks.

The author wishes to express his gratitude to Professors J. Appell, J.-P. Gossez, M. A. Krasnoselskij, V. S. Klimov, J. Mawhin, W. Petry, Ya. B. Rutitskij, I. V. Skrypnik, P. P. Zabrejko for their positive opinions on the private version of the present paper (as well as for their kind supports for all his research), which influenced strongly the shape of the submitted version.

The author is grateful for the constructive critical remark of one of the reviewers, which influenced the shape of the revised version.

Let us to inform that at the moment of acceptance of the paper the author succeeded in establishing some particular analogs of Theorems $1-2$ for the case of discontinuity in $u$ (or set-valuedness) of the nonlinear function $f(s, u)$; here for the construction of the "appropriate" Carathéodory relaxations $f_{n}$ [cf. (28)] we must use the approximate $C M$-selection theorems obtained by H. T. Nguyêñ, M.. Juniewicz and J. Ziemińska in December 1996. The author got also some new existence theorems for equation (1) in the resonance case of the strongly nonlinear pair $(L, F)$ via the modification of the topologic-approximate methods of the present paper; some different existence theorems in such a case were obtained via variational methods by A. Anane and J.-P. Gossez (1990) etc. All above results will be presented in forthcoming papers.

\section{References}

[1] Adams, R. T.: Sobolev Spaces. New York - London: Acad. Press 1975.

[2] Appell, J., Nguyên, H. T. and P. P. Zabrejko: General existence theorems for quasilinear elliptic systems without monotonicity. J. Math. Anal. Appl. 145 (1990), $26-38$.

[3] Appell, J., Nguyẽñ, H. T. and P. P. Zabrejko: Multivalued superposition operators in ideal spaces of vector functions. Part I. Indag. Math. (N.S.) 2 (1991), $385-395$.

[4] Appell, J., Nguyêñ, H. T. and P. P. Zabrejko: Multivalued superposition operators in ideal spaces of vector functions. Part II. Indag. Math. (N.S.) 2 (1991), $397-409$.

[5] Appell, J. and P. P. Zabrejko: Nonlinear Superposition Operators. Cambridge: Univ. Press 1990.

[6] Benkirane, A. and J.-P. Gossez: An approximation theorem in higher order Orlicz-Sobolev spaces and applications. Studia Math. 81 (1989), 231 - 255.

[7] Brezis, H. and F. E. Browder: Strongly nonlinear elliptic boundary value problems. Ann. Scu. norm. sup. Pisa 5 (1978), $587-603$. 
[8] Brezis, H. and F. E. Browder: Some properties of higher order Sobolev spaces. J. Math. Pures Appl. 61 (1982), 245 - 259.

[9] Browder, F. E.: Existence theory for boundary value problems for quasilinear elliptic systems with strongly nonlinear lower order terms. Proc. Symp. Pure Math. Amer. Math. Soc. 23 (1973), $269-286$.

[10] Browder, F. E.: Degree of mapping for nonlinear operators of monotone type: Strongly nonlinear mappings. Proc. Nat. Acad. Sci. U.S.A. 80 (1983), $2408-2409$.

(11) Dubinskij, Yu. A.: Nonlinear elliptic and parabolic equations (in Russian). Itohi Nauki i Tehniki VINITI (Modern problems in Math.) 9 (1976), 5 - 130.

[12] Dunford, N. and J. Schwartz: Linear operators. Vol. I: General Theory. New York: Intersci. 1957, and Leyden: Dordrecht 1963.

[13] Gossez, J.-P.: Nonlinear elliptic boundary value problems for equations with rapidly or slowly increasing coefficients. Trans. Amer. Math. Soc. 190 (1974), 163 - 205.

[14] J.-P. Gossez: Surjectivity results for pseudo-monotone mappings in complementary systems. J. Math. Anal. Appl. 53 (1976), $484-494$.

[15] Gossez, J.-P.: Some approximation properties in Orlicz-Sobolev spaces. Studia Math. 74 (1982), 17 - 24.

[16] Gossez, J.-P.: A strongly nonlinear elliptic problem in Orlicz-Sobolev spaces. Proc. Symp. Pure Math. Amer. Math. Soc. 45 (1986), 355 - 462.

[17] Gossez, J.-P. and V. Mustonen: Variational inequalities in Orlicz-Sobolev spaces. Nonlin. Anal. TMA 11 (1987), 379 - 392.

[18] Hess, P.: A strongly nonlinear elliptic boundary value problem. J. Math. Anal. Appl. 43 (1973), $241-249$.

[19] Klimov, V. S.: Functional inequalities and generalized capacities. Mat. Sbornik 187 (1996)1, $41-54$.

[20] Krasnoselskij, M. A.: Topological Methods in the Theory of Nonlinear Integral Equations (in Russian). Moscow: Gostehizdat 1956; Engl. transl.: New York: Macmillan 1964.

[21] Lions, J..L.: Quelques methods de résolution des problèmes aux limites non linéaires. Paris: Gauthies-Villars 1969.

[22] Luxemburg, W. A. J. and A. C. Zaanen: Riesz spaces. Vol. I. Amsterdam: North-Holland 1971.

[23] Mawhin, J.: Topological Degree and boundary valued problems for nonlinear differential equations. (Continuation principles and boundary value problems). Lect. Notes Math. 1537 (1993), $74-148$.

[24] Musielak, J.: Orlicz Spaces and Modular Spaces. Berlin: Springer-Verlag 1983.

[25] Nguyên, H. T.: Theory of semimodules of infra-semi-units in vector functions ideal spaces and its applications to integral operators (in Russian); Engl. transl. in: Soviet Math. Doklady 43 (1991), $615-619$.

[26] Nguyêñ, H. T.: Ideal Spaces of Vector Functions: Geometry, Interpolation and Applications to Nonlinear Operators and Equations. Habilitation Theses. Minsk: Byelorussian State Univ. 1991, pp. 1 - 341.

[27] Nguyèñ, H. T. and P. P.Zabrejko: ldeal spaces of vector-valued functions and their applications. In: Function Spaces (Teubner Texte zur Mathematik: Vol. 120; eds.: J. Musielak et al.). Stuttgart - Leipzig: Teubner 1991, pp. $112-119$.

[28] Petry: Systeme nichtlinearer Integralgleichungen mit positive definiten Kernen. Z. Angew. Math. Mech. (ZAMM) 47 (1967), 97 - 108. 
[29] Petryshyn, W. V.: Generalized Topological Degree and Semilinear Equations (Cambridge Tracts in Mathematics: Vol. 117). Cambridge: Univ. Press 1995.

[30] Rao, M. M. and Z. D. Ren: Theory of Orlicz Spaces. New York: Marcel Dekker Inc. 1991.

[31] Skrypnik, I. V.: Higher Order Nonlinear Elliptic Equations (in Russian). Kiev: Naukova Dumka 1973.

[32] Skrypnik, I. V.: Methods of Analysis of Nonlinear Elliptic Boundary Value Problems (in Russian). Moscow: Nauka 1990; Engl. transl.: Math. Monogr. AMS Vol. 139. Providence: Amer. Math. Soc. 1994.

[33] Väth, M.: Ideal Spaces. Lect. Notes Math. 1664 (1997), 1 - 146.

[34] Webb, J.: Boundary value problems for strongly nonlinear elliptic equations. J. London Math. Soc. 21 (1980), $128-132$.

[35] Zabrejko, P. P.: Schaefer's Method in theory of integral Hammerstein equations (in Russian). Mat. Sb. 13 (1971), 456 - 475; Engl. transl.: USSR Math. Sbornik 13 (1971), 451 -471 .

[36] Zabrejko, P. P.: Ideal spaces of vector functions (in Russian). Doklady Acad. Nauk BSSR. 31 (1987), 298 - 301.

[37] Zabrejko, P. P. and H. T. Nguyêñ: New Theorems on the solvability for operator and integral Hammerstein equations (in Russian). Diff. Uravnenia 27 (1991), 672 - 682; Engl. transl.: Diff. Equ. 27 (1991), 479 - 487.

[38] Zeidler, E.: Nonlinear Functional Analysis and its Applications. Parts 1 - 4. Berlin et al.: Springer-Verlag 1986 - 1996. 